\title{
THE CUSPIDAL MODULES OF THE FINITE GENERAL LINEAR GROUPS
}

\author{
D.I. DERIZIOTIS and C. P. GOTSIS
}

\begin{abstract}
In this paper we prove a conjecture due to R. Carter [2], concerning the action of the finite general linear group $G L_{n}(q)$ on a cuspidal module. As an application of this result, we work out the case $G L_{4}(q)$.
\end{abstract}

It is well known that the cuspidal characters of the finite groups of Lie type play a particularly important role in the complex representation theory of such groups. For the general linear group $\mathrm{GL}_{n}(q)$ over the field $\mathbf{F}_{q}$ of $q$ elements, these characters were determined by J. A. Green [5]. In the cases $n=2$ and $n=3$, the action of $\mathrm{GL}_{n}(q)$ on modules affording cuspidal characters has been studied in [9] and [8] respectively. In 1992 R. Carter [2] considered these cases using a different method from that used in [9] and [8]. The purpose of the present paper is to extend Carter's method to the general case, and to prove a conjecture stated in the introduction of [2]. This concerns the form of the entries of the matrices representing the action of $\mathrm{GL}_{n}(q)$ on a module affording a given cuspidal character.

This paper is organized as follows. In Section 1, we set up the basic definitions and notation from the representation theory needed here, and we determine a cuspidal module affording a given cuspidal character of $\operatorname{GL}_{n}(q)$. In Section 2, we do the work to obtain the action of $\mathrm{GL}_{n}(q)$ on such modules, and we discuss Carter's conjecture. In Section 3 we apply the general theory to the group $\mathrm{GL}_{4}(q)$. A considerable amount of detailed work was involved in the compilation of the results needed here to obtain the cuspidal matrices representing the generators of $\mathrm{GL}_{4}(q)$. This part of the work has not been included in the paper, since for our calculations we have followed more or less the same recipe as that used for the group $\mathrm{GL}_{3}(q)$ in [2].

\section{The cuspidal modules}

Throughout this paper, $G$ will denote the group $\operatorname{GL}_{n}(q), T$ the maximal torus of diagonal matrices in $G$, and $W$ the Weyl group with respect to $T$, which we shall identify with the subgroup of $G$ of permutation matrices. Then $T W$ is the subgroup $N$ of all monomial matrices in $G$.

We consider a maximal Coxeter torus $T_{n}$ of $G$. Then the elements of $T_{n}$ can be viewed as the diagonal matrices $\operatorname{diag}\left(t, t^{q}, \ldots, t^{q^{n-1}}\right), t \in \mathbf{F}_{q^{n}}^{*}$ where $\mathbf{F}_{q^{n}}^{*}$ denotes the multiplicative group of $\mathbf{F}_{q^{n}}$. Thus $T_{n} \cong \mathbf{F}_{q^{n}}^{*}$. It is well known (see [10]) that all the cuspidal characters have degree

$$
(q-1)\left(q^{2}-1\right) \ldots\left(q^{n-1}-1\right)
$$

and they are the Deligne-Lusztig irreducible characters $(-1)^{n-1} R_{T_{n}, \theta}$, where $\theta$ is a regular character of $T_{n}$ (see [1, Theorem 9.3.2]). 
Thus, counting the regular characters of $T_{n}$, it turns out from [7] that the group $G$ has

$$
\frac{1}{n} \sum_{d \mid n} \mu(d)\left(q^{n / d}-1\right)
$$

cuspidal characters in number, where $\mu$ is the Möbius function on the natural numbers.

Now, given a cuspidal character $\chi$ of $G$, we want to determine a module affording $\chi$. Such a module can be found inside the Gelfand-Graev module which is constructed as follows. We consider the root system $\Phi$ of $G$ with respect to $T$, and we fix a fundamental basis $\Delta=\left\{\alpha_{1}, \alpha_{2}, \ldots, \alpha_{n-1}\right\}$ in $\Phi$. Then a positive root $\alpha$ has the form

$$
\alpha=\sum_{i \leqslant k<j} \alpha_{k}, 1 \leqslant i<j \leqslant n .
$$

Let $X_{\alpha}$ be the root subgroup corresponding to the positive root $\alpha$. Then $X_{\alpha}$ consists of all upper unitriangular matrices $x_{\alpha}(\lambda), \lambda \in \mathbf{F}_{q}$, where the entry in the $(i, j)$-position is equal to $\lambda$ and all the other non-diagonal entries are equal to zero. The product

$$
\prod_{\alpha \in \Phi^{+}} X_{\alpha}
$$

is the upper unitriangular subgroup of $G$, where $\Phi^{+}$is the set of positive roots. Let $\psi$ be a non-trivial complex linear character of the additive group $\mathbf{F}_{q}^{+}$of $\mathbf{F}_{q}$. Since each $X_{\alpha}$ is isomorphic to $\mathbf{F}_{q}^{+}, \psi$ induces the linear character of $U$ (denoted also by $\psi$ ), given by

$$
\psi\left(\prod_{\alpha \in \Phi^{+}} x_{\alpha}\left(\lambda_{\alpha}\right)\right)=\prod_{\alpha \in \Delta} \psi\left(\lambda_{\alpha}\right) .
$$

The induced character $\psi^{G}$ is the so-called Gelfand-Graev character of $G$ which is afforded by the module $\mathbb{G} e$, where $e$ is the central primitive idempotent

$$
|U|^{-1} \sum_{u \in U} \psi\left(u^{-1}\right) u
$$

in the group algebra $\mathbb{C} U$ of $U$ over the field $\mathbb{C}$ of the complex numbers. One of the main properties of $\psi^{G}$ is that each of its irreducible components occurs with multiplicity one, and their number is $q^{n-1}(q-1)$. Moreover, every Deligne-Lusztig generalized character meets $\psi^{G}$ (see [1, Theorem 8.4.5]). In particular, every cuspidal character of $G$ occurs in $\psi^{G}$.

We recall that the Gelfand-Graev module is isomorphic to the $G$-module $\mathcal{F}$ of all $\mathbb{C}$ valued functions $f$ on $G$ defined by

$$
f(g u)=\psi\left(u^{-1}\right) f(g), \quad u \in U, \quad g \in G,
$$

where the $G$-action is given by

$$
(g f)(x)=f\left(g^{-1} x\right), \quad g, x \in G, \quad f \in \mathcal{F} .
$$

For $x \in G$ we define the function $f_{x}: G \rightarrow \mathbb{C}$ by

$$
f_{x}(x u)=\psi\left(u^{-1}\right), \text { for } u \in U
$$

and

$$
f_{x}(y)=0, \text { for } y \notin x U \text {. }
$$


Then these functions have the following properties:

Lemma 1. Let $x \in G$. Then (i) $f_{x} \in \mathcal{F}$, (ii) $g f_{x}=f_{g x}$, for all $g \in G$, and (iii) $f_{x u}=$ $\psi(u) f_{x}$, for all $u \in U$.

Proof. (i) For $g \in x U$ and $u \in U$ we have, $f_{x}(g u)=f_{x}\left(x u^{\prime} u\right)=\psi\left(u^{-1}\right) \psi\left(u^{\prime-1}\right)=$ $\psi\left(u^{-1}\right) f_{x}(g)$ where $g=x u^{\prime}, u^{\prime} \in U$. If $g \notin x U$ and $u \in U$ we have $f_{x}(g)=f_{x}(g u)=0$ as $g u \notin x U$. Thus $f_{x}(g u)=\psi\left(u^{-1}\right) f_{x}(g)=0$. (ii) For $y=g x u, u \in U$, we have $\left(g f_{x}\right)(y)=f_{x}\left(g^{-1} y\right)=\psi\left(u^{-1}\right)=f_{g x}(y)$, while for $y \notin g x U$ we have $f_{g x}(y)=0$ as well as $g f_{x}(y)=f_{x}\left(g^{-1} y\right)=0$, since $g^{-1} y \notin x U$. (iii) Let $y=x u^{\prime}, u^{\prime} \in U$. Then $f_{x u}(y)=f_{x u}\left(x u u^{-1} u^{\prime}\right)=\psi\left(u^{\prime}-1 u\right)=\psi(u) \psi\left(u^{\prime}-1\right)=\psi(u) f_{x}(y)$. If $y \notin x U$, then $\psi(u) f_{x}(y)=0$ and $f_{x u}(y)=0$ as $y \notin x u U$.

Corollary 2. $e f_{1}=f_{1}$.

Proof. We have $u f_{1}=f_{1 u}=\psi(u) f_{1}$, for all $u \in U$. Thus

$$
e f_{1}=|U|^{-1} \sum_{u \in U} \psi\left(u^{-1}\right) u f_{1}=|U|^{-1} \sum_{u \in U} \psi\left(u^{-1}\right) \psi(u) f_{1}=f_{1} \text {. }
$$

We next consider an irreducible component $\chi$ of $\psi^{G}$ and let $\varepsilon_{\chi}$ be the corresponding central primitive idempotent of $\mathbb{C} G$. Then the $G$-module $\varepsilon_{\chi} \mathcal{F}$ is the unique irreducible $G$-submodule of $\mathcal{F}$ affording $\chi$ and so $\varepsilon_{\chi} \mathcal{F}$, as $U$-module, affords the character $\left.\chi\right|_{U}$. By the Frobenius reciprocity theorem, we have

$$
\left(\left.\chi\right|_{U}, \psi\right)=\left(\chi, \psi^{G}\right)=1 .
$$

Therefore the $U$-module $\varepsilon_{\chi} \mathcal{F}$ contains a unique 1-dimensional submodule affording $\psi$. Since $u\left(\varepsilon_{\chi} f_{1}\right)=\varepsilon_{\chi}\left(u f_{1}\right)=\psi(u) \varepsilon_{\chi} f_{1}, u \in U$, the subspace $\mathbb{C} \varepsilon_{\chi} f_{1}$ of $\varepsilon_{\chi} \mathcal{F}$ is the required one, and we have $\varepsilon_{\chi} \mathcal{F}=\mathbb{C} G \varepsilon_{\chi} f_{1}$. The non-zero multiples of the vector $\varepsilon_{\chi} f_{1}$ are called Bessel vectors for the character $\chi$.

Let $\chi$ be a cuspidal character. Following [2], to determine the action of $G$ on the Bessel vector $\varepsilon_{\chi} f_{1}$, we write $\varepsilon_{\chi} f_{1}$ in terms of a basis of $\mathcal{F}$ which is obtained by considering the left cosets of $U$ in $G$. These are determined by the Bruhat decomposition

$$
G=\bigcup_{w \in W} U_{w^{-1}} T w U
$$

where $U_{w^{-1}}=U \cap w w_{0} U w_{0} w^{-1}, w_{0}$ being the element of maximal length in $W$. Thus the set $X=\bigcup_{w \in W} U_{w^{-1}} T w$ is a complete set of left coset representatives of $U$ in $G$, and every element $f \in \mathcal{F}$ can be expressed uniquely in the form $f=\sum_{x \in X} f(x) f_{x}$. In other words, the set $\left\{f_{x} \mid x \in X\right\}$ is a basis of $\mathcal{F}$, and we call this basis the "Bruhat basis" of $\mathcal{F}$. In order to express $\varepsilon_{\chi} f_{1}$ as a linear combination of the basis elements $f_{x}, x \in X$, we need to recall [3] a basic fact concerning the relation of the central primitive idempotents of $\mathbb{C} G$ and those of the Hecke algebra $\mathscr{H}=e \mathbb{C} G e$ of $\psi^{G}$. We put ${ }^{g} U=g U g^{-1}, U_{g}^{+}=U \cap^{g^{-1}} U$, for $g \in G$, and we let

$$
N_{\psi}=\left\{n \in N \mid{ }^{n} \psi(u)=\psi(u), u \in U_{n^{-1}}^{+}\right\}
$$


where ${ }^{n} \psi$ is the character of ${ }^{n} U$ given by ${ }^{n} \psi\left(n u n^{-1}\right)=\psi(u), u \in U$. Then the elements $a_{n}=\left|U: U_{n^{-1}}^{+}\right|$ene, $n \in N_{\psi}$, form a basis for $\mathscr{H}$. Since $\psi^{G}$ is multiplicity-free, $\mathcal{H}$ is a commutative algebra, and hence a direct sum of 1-dimensional subalgebras. The primitive idempotents of $\mathscr{H}$ are precisely the elements $e_{\chi}=\varepsilon_{\chi} e$ for all central primitive idempotents $\varepsilon_{\chi}$ of $\mathbb{C} G$ with $\left(\chi, \psi^{G}\right)=1$, and we have

$$
e_{\chi}=\frac{\chi(1)}{|G: U|} \sum_{n \in N_{\psi}}\left|U: U_{n^{-1}}^{+}\right|^{-1} \chi\left(\left|U: U_{n^{-1}}^{+}\right| e n^{-1} e\right)\left|U: U_{n^{-1}}^{+}\right| \text {ene }
$$

(see [3], §11D). Thus Corollary 2 and Lemma 1 (ii) give

$$
e_{\chi} f_{1}=\frac{\chi(1)}{|G: U|} \sum_{n \in N_{\psi}}\left|U: U_{n^{-1}}^{+}\right| \chi\left(e n^{-1} e\right) e f_{n} .
$$

Therefore we have to express $e f_{n}, n \in N_{\psi}$, in terms of the elements $f_{x}, x \in X$.

Proposition 3. Let $n \in N_{\psi}$. Then

$$
e f_{n}=\frac{1}{q^{\ell(w)}} \sum_{u \in U_{w^{-1}}} \psi\left(u^{-1}\right) f_{u n}
$$

where $n=t w, t \in T, w \in W$, and $\ell(w)$ denotes the length of $w$.

Proof. We recall that if $w \in W$, then $U=U_{w} U_{w}^{+}=U_{w}^{+} U_{w}$ and $U_{w} \cap U_{w}^{+}=1$. Thus for $u \in U$ we may write $u=u_{1} u_{2}, u_{1} \in U_{w^{-1}}$ and $u_{2} \in U_{w^{-1}}^{+}$, and we have

$$
\begin{aligned}
e f_{n} & =|U|^{-1} \sum_{u \in U} \psi\left(u^{-1}\right) f_{u n} \\
& =|U|^{-1} \sum_{u_{1} \in U_{w^{-1}}} \sum_{u_{2} \in U_{w^{-1}}} \psi\left(u_{1}^{-1}\right) \psi\left(u_{2}^{-1}\right) f_{u_{1} u_{2} n} \\
& =|U|^{-1} \sum_{u_{1} \in U_{w^{-1}}} \sum_{u_{2} \in U_{w^{-1}}^{+}} \psi\left(u_{1}^{-1}\right) \psi\left(u_{2}^{-1}\right) f_{u_{1} n\left(n^{-1} u_{2} n\right)} \\
& =|U|^{-1} \sum_{u_{1} \in U_{w^{-1}}} \sum_{u_{2} \in U_{w^{-1}}^{+}} \psi\left(u_{1}^{-1}\right) \psi\left(u_{2}^{-1}\right) \psi\left(n^{-1} u_{2} n\right) f_{u_{1} n}
\end{aligned}
$$

by Lemma 1 (iii) as $n^{-1} u_{2} n \in U \cap^{n^{-1}} U$. Since $n \in N_{\psi}$, we have $\psi\left(n^{-1} u_{2} n\right)={ }^{n} \psi\left(u_{2}\right)=$ $\psi\left(u_{2}\right)$. Thus

$$
\begin{aligned}
e f_{n} & =|U|^{-1} \sum_{u_{1} \in U_{w^{-1}}} \sum_{u_{2} \in U_{w^{+}}^{+1}} \psi\left(u_{1}^{-1}\right) \psi\left(u_{2}^{-1}\right) \psi\left(u_{2}\right) f_{u_{1} n} \\
& =|U|^{-1}\left(\sum_{u_{2} \in U_{w^{-1}}^{+}} \psi\left(u_{2}^{-1}\right) \psi\left(u_{2}\right)\right) \sum_{u_{1} \in U_{w^{-1}}} \psi\left(u_{1}^{-1}\right) f_{u_{1} n} \\
& =|U|^{-1} \mid U_{w^{-1}}^{+} \sum_{u_{1} \in U_{w^{-1}}} \psi\left(u_{1}^{-1}\right) f_{u_{1} n} \\
& =q^{-\ell(w)} \sum_{u_{1} \in U_{w^{-1}}} \psi\left(u_{1}^{-1}\right) f_{u_{1} n},
\end{aligned}
$$


as $|U|=q^{\ell\left(w_{0}\right)}$ and $\left|U_{w^{-1}}^{+}\right|=q^{\ell\left(w_{0}\right)-\ell(w)}$.

Corollary 4. $e_{\chi} f_{1}=\frac{\chi(1)}{|G: U|} \sum_{n \in N_{\psi}} \sum_{u \in U_{w^{-1}}} \chi\left(e n^{-1} e\right) \psi\left(u^{-1}\right) f_{u n}$,

where in the second sum the element $w$ of $W$ is such that $n \in T w$, for $n \in N_{\psi}$.

\section{The G-action on the cuspidal modules}

Let $\chi$ be a cuspidal character of $G$. From now on we shall take as a Bessel vector the vector $b=|G: U| \chi(1)^{-1} e_{\chi} f_{1}=\left(q^{n}-1\right) e_{\chi} f_{1}$. Since $U_{1}=1$ and if $n=1$ then $w=1$, we have

$$
b=f_{1}+\sum_{\substack{n \in N_{\psi} \\ n \neq 1}} \sum_{u \in U_{w^{-1}}} \chi\left(e n^{-1} e\right) \psi\left(u^{-1}\right) f_{u n}
$$

as $\chi(e)=1$. This is the unique Bessel vector that has the coefficient of $f_{1}$ equal to 1 .

Now to determine the action of $G$ on the cuspidal module $\mathbb{C} G b$, we consider as in [2] the affine subgroup $A$ of $G$ consisting of all matrices $\left(a_{i j}\right)$ in $G$ with $a_{n j}=0$ for $j=1,2, \ldots, n-1$ and $a_{n n}=1$. Then $G$ is generated by $A$ and the permutation matrix

$$
s:=s_{n-1}=\left(\begin{array}{ccccc}
1 & & & & O \\
& \ddots & & & \\
& & 1 & & \\
& & & 0 & 1 \\
O & & & 1 & 0
\end{array}\right) .
$$

We also consider the subgroup $G_{0}$ of $A$ consisting of all matrices $\left(a_{i j}\right) \in A$ with $a_{i n}=0$, $i=1,2, \ldots, n-1$.

Lemma 5. If $Y$ is a set of left coset representatives of the subgroup $U_{0}=G_{0} \cap U$ in $G_{0}$, then $Y$ is also a set of left coset representatives of $U$ in $A$.

Proof. We have $A=G_{0} U_{\sigma}^{+}$and $U=U_{0} U_{\sigma}^{+}$where

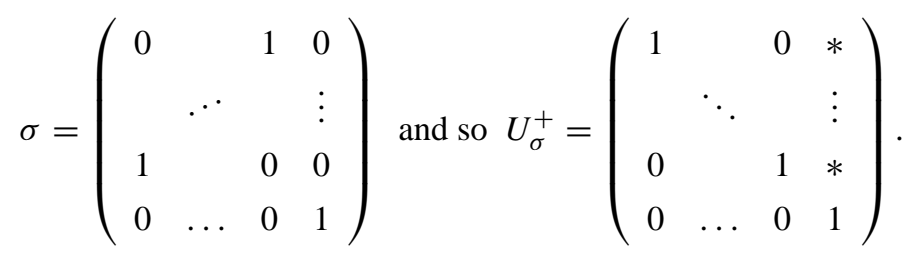

Since $G_{0} \cap U_{\sigma}^{+}=1$, we have $A=\bigcup_{y \in Y} y U_{0} U_{\sigma}^{+}=\bigcup_{y \in Y}^{\cdot} y U$ as required.

Let $W_{0}=\left\{w \in W \mid w \in G_{0}\right\}$ and $T_{0}=T \cap A$. Then we have the Bruhat decomposition of $G_{0}$

$$
G_{0}=\bigcup_{w \in W_{0}} U_{w^{-1}} T_{0} w U_{0}
$$


and so by Lemma 5, we may take the set $Y=\bigcup_{w \in W_{0}} U_{w^{-1}} T_{0} w$ as a set of left coset representatives of $U$ in $A$. We show now that the vectors $y b, y \in Y$, form a basis of the cuspidal module $\mathbb{C} G b$. For this we need first to determine the matrix form of the elements in $N_{\psi}$.

Lemma 6. The set $N_{\psi}$ consists of the monomial matrices of the form

$$
\left(\begin{array}{ccc}
0 & & t_{1} I_{n_{1}} \\
& . \cdot & \\
t_{k} I_{n_{k}} & & 0
\end{array}\right), t_{1}, \ldots, t_{k} \in \mathbf{F}_{q}^{*},
$$

where $\left(n_{1}, \ldots, n_{k}\right)$ is an unordered partition of $n$ and $I_{n_{i}}$ denotes the identity matrix of degree $n_{i}$.

Proof. Let $n=t w, t \in T, w \in W$. We show first that $n \in N_{\psi}$ if and only if $w\left(\Phi^{+}\right) \cap \Delta=$ $w(\Delta) \cap \Phi^{+}$and $w(\alpha)(t)=1$ for all $\alpha \in w^{-1}(\Delta) \cap \Phi^{+}$. We have

$$
U \cap{ }^{n} U=\prod_{\substack{\alpha \in \Phi^{+} \\ w(\alpha) \in \Phi^{+}}} X_{w(\alpha)}
$$

Let $u=\prod_{\substack{\alpha \in \Phi^{+} \\ w(\alpha) \in \Phi^{+}}} x_{w(\alpha)}\left(\lambda_{\alpha}\right) \in U \cap{ }^{n} U$. Then $\psi(u)=\prod_{\substack{\alpha \in \Phi^{+} \\ w(\alpha) \in \Delta}} \psi\left(\lambda_{\alpha}\right)$ and ${ }^{n} \psi(u)=\psi\left(u^{n}\right)=$ $\prod \psi\left(w(\alpha)\left(t^{-1}\right) \lambda_{\alpha}\right)$. Thus $n \in N_{\psi}$ if and only if

$$
\prod_{\alpha \in \Phi^{+} \cap w^{-1}(\Delta)} \psi\left(\lambda_{\alpha}\right)=\prod_{\alpha \in \Delta \cap w^{-1}\left(\Phi^{+}\right)} \psi\left(w(\alpha)\left(t^{-1}\right) \lambda_{\alpha}\right)
$$

for any choice of $\lambda_{\alpha} \in \mathbf{F}_{q}^{+}, \alpha \in \Phi^{+}$. If there were $\alpha \in \Phi^{+} \cap w^{-1}(\Delta)$ with $\alpha \notin w^{-1}\left(\Phi^{+}\right) \cap \Delta$, then taking $\lambda_{\alpha} \in \mathbf{F}_{q}^{+}$with $\psi\left(\lambda_{\alpha}\right) \neq 1$ and $\lambda_{\beta}=0, \beta \neq \alpha, \beta \in\left(\Phi^{+} \cap w^{-1}(\Delta)\right) \cup$ $\left(\Delta \cap w^{-1}\left(\Phi^{+}\right)\right)$, we would have $\psi\left(\lambda_{\alpha}\right)=1$ by (1), a contradiction. Similarly, if $\alpha \in$ $w^{-1}\left(\Phi^{+}\right) \cap \Delta$ then $\alpha \in \Phi^{+} \cap w^{-1}(\Delta)$. Thus $\Phi^{+} \cap w^{-1}(\Delta)=w^{-1}\left(\Phi^{+}\right) \cap \Delta$. That is, $w\left(\Phi^{+}\right) \cap \Delta=\Phi^{+} \cap w(\Delta)$. Moreover, if $w(\alpha)\left(t^{-1}\right) \neq 1$ for some $\alpha \in \Phi^{+} \cap w^{-1}(\Delta)$, taking $\lambda_{\alpha} \in \mathbf{F}_{q}^{+}$with $\psi\left(\left(w(\alpha)\left(t^{-1}\right)-1\right) \lambda_{\alpha}\right) \neq 1$ and $\lambda_{\beta}=0, \beta \neq \alpha, \beta \in \Phi^{+} \cap w^{-1}(\Delta)$, we have by (1), $\psi\left(\lambda_{\alpha}\right)=\psi\left(w(\alpha)\left(t^{-1}\right) \lambda_{\alpha}\right)$ or $\psi\left(\left(w(\alpha)\left(t^{-1}\right)-1\right) \lambda_{\alpha}\right)=1$, a contradiction. This shows that $w(\alpha)\left(t^{-1}\right)=1$, and so $w(\alpha)(t)=\left(w(\alpha)\left(t^{-1}\right)\right)^{-1}=1$. The converse is obvious.

We identify now $\Phi$ with the root system $\left\{e_{i}-e_{j} \mid 1 \leqslant i, j \leqslant n\right.$ and $\left.i \neq j\right\}$ in $\mathbb{R}^{n}$ where $\left\{e_{1}, \ldots, e_{n}\right\}$ is the normal basis in $\mathbb{R}^{n}$. Let $\Delta=\left\{e_{i}-e_{i+1} \mid 1 \leqslant i \leqslant n-1\right\}$. Suppose that $t$ and $w$ have the form

$$
t=\left(\begin{array}{ccc}
t_{1} I_{n_{1}} & & 0 \\
& \ddots & \\
0 & & t_{k} I_{n_{k}}
\end{array}\right) \text { and } w=\left(\begin{array}{ccc}
0 & & I_{n_{1}} \\
& \ddots & \\
I_{n_{k}} & & 0
\end{array}\right) \text {. }
$$

Then it is straightforward to see that $w\left(\Phi^{+}\right) \cap \Delta=w(\Delta) \cap \Phi^{+}$and $w(\alpha)(t)=1$ for all $\alpha \in w^{-1}(\Delta) \cap \Phi^{+}$. Conversely, suppose that $w$ and $t$ satisfy this condition and let $\pi$ be the 
permutation that corresponds to $w$. We have $w\left(\Phi^{+}\right) \cap \Delta=\left\{e_{\pi(i)}-e_{\pi(j)} \mid 1 \leqslant i<j \leqslant n\right.$ and $\pi(j)=\pi(i)+1\}$ and $w(\Delta) \cap \Phi^{+}=\left\{e_{\pi(i)}-e_{\pi(i+1)} \mid 1 \leqslant \pi(i)<\pi(i+1) \leqslant n\right\}$. Since $w\left(\Phi^{+}\right) \cap \Delta=\Phi^{+} \cap w(\Delta), \pi$ must satisfy the condition: $\pi(i+1)=\pi(i)+1$ whenever $\pi(i)<\pi(i+1), 1 \leqslant i \leqslant n-1$. If $\delta_{\rho, k}$ denotes the Kronecker delta, then $w=\left(\delta_{i, \pi(j)}\right)$. Let $\pi\left(j_{1}\right)=1$. As $\pi\left(j_{1}\right)<\pi\left(j_{1}+1\right)$, we have $\pi\left(j_{1}+1\right)=\pi\left(j_{1}\right)+1=2$ and inductively $\pi\left(j_{1}+k\right)=k+1,0 \leqslant k \leqslant n-j_{1}$. That is, $\delta_{i, \pi\left(n-n_{1}+i\right)}=1,1 \leqslant i \leqslant n_{1}$, where $n_{1}=n-j_{1}+1$. In the same way, if $\pi\left(j_{2}\right)=n-j_{1}+2=n_{1}+1$, then $\pi\left(j_{2}\right)<\pi\left(j_{2}+1\right)$ and so $\pi\left(j_{2}+1\right)=\pi\left(j_{2}\right)+1=n_{1}+2$ and inductively $\pi\left(j_{2}+k\right)=n_{1}+1+k$, $0 \leqslant k \leqslant j_{1}-j_{2}-1$. That is, $\delta_{n_{1}+i, \pi\left(n-n_{1}-n_{2}+i\right)}=1,1 \leqslant i \leqslant n_{2}$, where $n_{2}=j_{1}-j_{2}$. Therefore, an easy induction argument gives that $w$ has the required form.

Now as $w(\alpha)(t)=1, \alpha \in w^{-1}\left(\Phi^{+}\right) \cap \Delta$, we have $\alpha(t)=1, \alpha \in \Phi^{+} \cap w(\Delta)$. But $\alpha(t)=\lambda_{i} \lambda_{j}^{-1}$, where $t=\operatorname{diag}\left(\lambda_{1}, \ldots, \lambda_{n}\right)$ and $\alpha=e_{i}-e_{j}, 1 \leqslant i, j \leqslant n$. In our case $\alpha$ is one of the roots $e_{\pi(i)}-e_{\pi(i+1)}$, where $\pi$ is the corresponding permutation. Thus $t_{\pi(i)}=t_{\pi(i+1)}=t_{\pi(i)+1}$ whenever $\pi(i)<\pi(i+1)$. That is, $\lambda_{1}=\ldots=\lambda_{n_{1}}, \lambda_{n_{1}+1}=$ $\ldots=\lambda_{n_{1}+n_{2}}, \ldots, \lambda_{n_{1}+\ldots+n_{k-1}+1}=\ldots=\lambda_{n}$. This shows that $t$ must have the required form, where $t_{1}=\lambda_{1}, t_{2}=\lambda_{n_{1}+1}, \ldots, t_{k}=\lambda_{n_{1}+\ldots+n_{k-1}+1}$.

From this Lemma one can obtain the dimension of the Hecke algebra $\mathscr{H}$.

Corollary 7. $\left|N_{\psi}\right|=q^{n-1}(q-1)$.

Proof. We consider the elements

$$
\left(\begin{array}{cc}
0 & t_{1} I_{k} \\
t_{2} I_{n-k} & 0
\end{array}\right), \quad 1 \leqslant k \leqslant n-1
$$

in $N_{\psi}$. Replacing for a given $k$ the block $t_{2} I_{n-k}$ by any element of the corresponding set in $\mathrm{GL}_{n-k}(q)$, we obtain by induction $q^{n-k-1}(q-1)^{2}$ elements in $N_{\psi}$. Doing this for any $k, 1 \leqslant k \leqslant n-1$, we obtain $(q-1)^{2} \sum_{k=1}^{n-1} q^{n-k-1}=(q-1)\left(q^{n-1}-1\right)$ elements in $N_{\psi}$. The only elements of $N_{\psi}$ that have not been considered are those in $Z(G)$. Thus $\left|N_{\psi}\right|=(q-1)\left(q^{n-1}-1\right)+(q-1)=q^{n-1}(q-1)$.

Corollary 8. $N_{\psi} \cap A=1$.

Proof. The proof is obvious.

Proposition 9. The set $\{y b \mid y \in Y\}$ is a basis of the cuspidal module $\mathbb{C} G b$.

Proof. The set $N_{0}=\bigcup_{w \in W_{0}} T_{0} w$ is a set of double coset representatives of $U$ in $A$. We consider the induced character $\psi^{A}$ of $A$, where $\psi$ is the character of $U$ considered before. Applying Mackey's theorem on $\psi^{A}$ we have

$$
\left(\psi^{A}, \psi^{A}\right)=\sum_{n \in N_{0}}\left(\psi^{n}, \psi\right)_{U_{n^{-1}}^{+}}=1
$$


as $\psi \neq{ }^{n} \psi$ on $U_{n^{-1}}^{+}, n \in N_{0}$ unless $n=1$ by Corollary 8 . That is, the character $\psi^{A}$ is irreducible. Let $\chi$ be any cuspidal character of $G$, and let $\left.\chi\right|_{A}$ be its restriction on $A$. By Frobenius reciprocity we have

$$
\left(\left.\chi\right|_{A}, \psi^{A}\right)_{A}=\left(\chi, \psi^{G}\right)_{G}=1 .
$$

Since $\psi^{A}$ is an irreducible character of $A$ and $\left.\operatorname{deg} \chi\right|_{A}=\operatorname{deg} \psi^{A}$, we must have $\psi^{A}=\left.\chi\right|_{A}$ for any cuspidal character $\chi$ of $G$. Therefore, if $\chi$ is a given cuspidal character of $G$, the $G$-module $\mathbb{C} G b$ affording $\chi$ remains irreducible as an $A$-module, since this affords $\left.\chi\right|_{A}$. On the other hand, the $A$-module $\mathbb{C} A b$ is a non-zero $A$-submodule of the irreducible $A$-module $\mathbb{C} G b$. Thus $\mathbb{C} A b=\mathbb{C} G b$. Now if $a \in A$, then $a=y u$ for some $y \in Y$ and $u \in U$. Thus $a b=\psi(u) y b$. This means that the elements $y b, y \in Y$, generate the space $\mathbb{C} G b$. But $|Y|=\operatorname{dim} \mathbb{C} G b$, and so the set $\{y b \mid y \in Y\}$ is a basis of $\mathbb{C} G b$ as required.

To investigate the action of $G$ on $\mathbb{C} G b$ it is sufficient to obtain the action of $A$ and that of the element $s=s_{n-1}$ on the basis $\{y b \mid y \in Y\}$. But if $a \in A, y \in Y$, then by the Bruhat decomposition, we have $a=y^{\prime} u$ for some $y^{\prime} \in Y$ and $u \in U$. Therefore we have $a(y b)=y^{\prime} u b=\psi(u) y^{\prime} b$. In other words, the action of $A$ is monomial with respect to the above basis. Thus it remains to investigate the action of the element $s$ on the vectors $y b$, $y \in Y$.

Let $X_{\psi}=\left\{u n \mid n \in N_{\psi}, u \in U_{w^{-1}}\right.$, where $\left.n \in T w\right\}$.

We put $\xi_{a}=\chi\left(e n^{-1} e\right) \psi\left(u^{-1}\right)$, where $a=u n \in X_{\psi}$. We then have

$$
b=\sum_{a \in X_{\psi}} \xi_{a} f_{a} .
$$

The coefficients $\xi_{a}$ are usually called "Bessel coefficients". Thus for $y \in Y$, we have by Lemma 1 (ii)

$$
y b=\sum_{a \in X_{\psi}} \xi_{a} f_{y a}=\sum_{z \in Z} \xi_{z} f_{y z}+\sum_{a \in X_{\psi} \backslash Z} \xi_{a} f_{y a}
$$

where $Z=Z(G)$. For $a \in X_{\psi}, y \in Y$, we let $s y a=x_{\text {sya }} v_{\text {sya }}$ be the Bruhat decomposition of sya, where $x_{\text {sya }} \in X$ and $v_{\text {sya }} \in U$. With this notation we have by Lemma 1 (iii)

$$
s(y b)=\sum_{z \in Z} \xi_{z} \psi\left(v_{s y z}\right) f_{x_{s y z}}+\sum_{a \in X_{\psi} \backslash Z} \xi_{a} \psi\left(v_{s y a}\right) f_{x_{s y a}} .
$$

We notice that in (2) if $a, a^{\prime} \in X_{\psi} \backslash Z$, then $x_{s y a}=x_{s y a^{\prime}}$ implies $a=a^{\prime}$. For, if $a=u n$ and $a^{\prime}=u^{\prime} n^{\prime}$, then $u n v_{\text {sya }}^{-1}=u^{\prime} n^{\prime} v_{\text {sya }}^{-1}$ and from the uniqueness of the Bruhat decomposition we get $a=a^{\prime}$.

Next, we express the vector $s(y b)$ as a linear combination of the basis elements $y^{\prime} b$, $y^{\prime} \in Y$. We have

$$
s(y b)=\sum_{y^{\prime} \in Y} \lambda_{y, y^{\prime}}\left(y^{\prime} b\right)=\sum_{z \in Z} \sum_{y^{\prime} \in Y} \lambda_{y, y^{\prime}} \xi_{z} f_{y^{\prime} z}+\sum_{a \in X_{\psi} \backslash Z} \sum_{y^{\prime} \in Y} \lambda_{y, y^{\prime}} \xi_{a} f_{y^{\prime} a} .
$$

For this expression (3) of $s(y b)$ we notice that the vectors $f_{y^{\prime} z}, y^{\prime} \in Y, z \in Z$, belong to the Bruhat basis of the $G$-module $\mathcal{F}$, and $y^{\prime} z=y^{\prime \prime} z^{\prime}, y^{\prime}, y^{\prime \prime} \in Y, z, z^{\prime} \in Z$ if and only if $y^{\prime}=y^{\prime \prime}$ and $z=z^{\prime}$. Also, since $A \cap N_{\psi}=1$ the vectors $f_{y^{\prime} a}, a \in X_{\psi} \backslash Z, y^{\prime} \in Y$, are multiples of the Bruhat basis elements $f_{g}, g \in X$, with $g \notin Y Z$. 
Now we compare the two expressions (2) and (3) of $s(y b)$. Since, for any $z^{\prime} \in Z$ the elements $s y z^{\prime}$ are not in $A Z$ (and so $x_{s y z} \notin Y Z$ ), we see that there is no $z^{\prime} \in Z$ for which $f_{y^{\prime} z}=f_{x y z^{\prime}}$ for some $z \in Z$ and $y^{\prime} \in Y$. Therefore we must have

$$
\sum_{z \in Z} \sum_{y^{\prime} \in Y} \lambda_{y, y^{\prime}} \xi_{z} f_{y^{\prime} z}=\sum_{a \in X_{\psi} \backslash Z} \xi_{a} \psi\left(v_{s y a}\right) f_{x_{s y a}} .
$$

Now if we have an $a \in Z_{\psi}$ such that sya $\in A$, then $x_{s y a} z=x_{s y(a z)}$ and $v_{s y a}=v_{s y(a z)}$ for all $z \in Z$. Thus we obtain

$$
\sum_{z \in Z} \sum_{y^{\prime} \in Y} \lambda_{y, y^{\prime}} \xi_{z} f_{y^{\prime} z}=\sum_{z \in Z} \sum_{\substack{a \in X_{\psi} \backslash Z \\ x_{s y a} \in Y}} \xi_{a z} \psi\left(v_{s y a}\right) f_{x_{s y(a z)}} .
$$

The equation 4 says that if we put $Y^{\prime}=\left\{y^{\prime} \in Y \mid y^{\prime}=x_{\text {sya }}\right.$ for some $\left.a \in X_{\psi} \backslash Z\right\}$, then for $y^{\prime} \in Y^{\prime}$ we have $\lambda_{y, y^{\prime}}=\xi_{a} \psi\left(v_{s y a}\right)=\xi_{z}^{-1} \xi_{a z} \psi\left(u_{s y a}\right)$, for all $z \in Z$, where $a$ is the element of $X_{\psi} \backslash Z$ such that $y^{\prime}=x_{s y a}$. Otherwise, if $y^{\prime} \in Y \backslash Y^{\prime}$, then $\lambda_{y, y^{\prime}}=0$. Thus, given $y, y^{\prime} \in Y$, we consider the element $y^{-1} s y^{\prime}$ and its Bruhat decomposition $y^{-1} s y^{\prime}=u t w v^{-1}$, $w \in W, t \in T, u \in U_{w^{-1}}, v \in U$. Then if $u t w \in X_{\psi} \backslash Z$, we have

$$
\lambda_{y, y^{\prime}}=\xi_{u t w} \psi(v) \text {. }
$$

Otherwise $\lambda_{y, y^{\prime}}=0$.

Now if $\lambda_{y, y^{\prime}}=\xi_{u t w} \psi(v)$, then $w \neq 1$; otherwise, $u$ would be 1 and so by Lemma 6 , $t \in Z$, a contradiction, since $u t w \notin Z$. Thus $w_{0} w^{-1} \neq w_{0}$, and so there is a simple root $\alpha \in \Delta$ such that $w_{0} w^{-1}(\alpha)=\beta \in \Phi^{+}$. Therefore we have ${ }^{w w_{0}} X_{\beta}=X_{w w_{0}(\beta)}=X_{\alpha} \subseteq$ $U \cap{ }^{w w_{0}} U=U_{w^{-1}}$. Now we may choose an element $u_{1} \in X_{\alpha}$ such that $\psi(v)=\psi\left(u_{1}\right)$. This allows us to write $\xi_{a} \psi(v)=\xi_{a} \psi\left(u_{1}\right)=\chi\left(e n^{-1} e\right) \psi\left(u^{-1}\right) \psi\left(u_{1}\right)=\xi_{u_{1}^{-1} u n}$, where $a=u n, n=t w$, sya $=y^{\prime} v$. In other words, the coefficient $\lambda_{y, y^{\prime}}$ is one of the Bessel coefficients, namely the coefficient $\xi_{u_{1}^{-1} u n}$ (which is equal to $\xi_{u^{\prime}-1}$ un for any other choice $u^{\prime} \in U_{w^{-1}}$ such that $\left.\psi\left(u^{\prime}\right)=\psi(v)\right)$. This proves Carter's conjecture [2], which we state now as a theorem.

Theorem 10. Let $R$ be the matrix representation corresponding to the cuspidal module $\mathbb{C} G b$ with respect to the basis $\{y b \mid y \in Y\}$. Then every non-zero entry of the matrix $R(s)=\left(\lambda_{y, y^{\prime}}\right)_{y, y^{\prime} \in Y}$ is a Bessel coefficient.

More precisely, if $y^{-1} s y^{\prime}=u t w v$ is the Bruhat decomposition of the element $y^{-1} s y^{\prime}$ for $y, y^{\prime} \in Y$, then $\lambda_{y, y^{\prime}}=\xi_{a}$ for any a of the form $u_{1}$ utw $\in X_{\psi} \backslash Z$ where $u_{1} \in U_{w^{-1}}$ such that $\psi\left(u_{1}\right)=\psi(v)$. Otherwise $\lambda_{y, y^{\prime}}=0$. Moreover

$$
\lambda_{y, y^{\prime}}=\xi_{z}^{-1} \xi_{a z}(\text { for every } z \in Z) \text {. }
$$

As a result of the above Theorem 10, one can prove Proposition 24 in [2].

Corollary 11. Let $y, y^{\prime} \in Y$. Then $\lambda_{y, y^{\prime}}=\overline{\lambda_{y^{\prime}, y}}$. In other words, the matrix $S$ representing the action of $s$ on $\mathbb{C} G b$ is Hermitian. 
Proof. We may suppose that $\lambda_{y, y^{\prime}} \neq 0$. Then there exist $a \in X_{\psi} \backslash Z$ such that $s y a=$ $y^{\prime} z v \in A Z, z \in Z, v \in U$. Without loss of generality, we may take $z=1$. Then we have $\lambda_{y, y^{\prime}}=\xi_{a} \psi(v)$. Let $a=u n, n \in N_{\psi} \backslash Z, u \in U_{w^{-1}}$, where $n=t w, t \in T, w \in W$. Therefore we have

$$
\lambda_{y, y^{\prime}} \xi_{n} \psi\left(u^{-1}\right) \psi(v)
$$

By writing $v=v_{1} v_{2}, v_{1} \in U_{w}, v_{2} \in U_{w}^{+}$, we have $y u=s y^{\prime} v_{1} v_{2} n^{-1}=s y^{\prime} v_{1} n^{-1}\left(n v_{2} n^{-1}\right)$. But $v_{1} n^{-1} \in X_{\psi} \backslash Z$, as $v_{1} \in U_{\left(w^{-1}\right)^{-1}}$, and $n v_{2}^{-1} n^{-1} \in{ }^{w} U \cap U \subseteq U$. Thus we have sy $a^{\prime}=y v^{\prime} \in A$, where $a^{\prime}=v_{1} n^{-1} \in X_{\psi} \backslash Z$ and $v^{\prime}=u n v_{2}^{-1} n^{-1} \in U$.

Therefore $\lambda_{y^{\prime}, y}=\xi_{a^{\prime}} \psi\left(v^{\prime}\right)=\xi_{n^{-1}} \psi\left(v_{1}^{-1}\right) \psi(u) \psi\left(n v_{2}^{-1} n^{-1}\right)=\left(\right.$ as $n v_{2}^{-1} n^{-1} \in U \cap$ ${ }^{n} U$ and $\psi={ }^{n} \psi$ on $\left.U \cap{ }^{n} U\right)=\xi_{n^{-1}} \psi(u) \psi\left(v_{1}^{-1}\right)^{n} \psi\left(n v_{2}^{-1} n^{-1}\right)=\bar{\xi}_{n} \overline{\psi\left(u^{-1}\right)} \overline{\psi\left(v_{1}\right)} \overline{\psi\left(v_{2}\right)}$ $=\overline{\xi_{n} \psi\left(u^{-1}\right) \psi(v)}=\overline{\lambda_{y^{\prime}, y}}$ since $\xi_{n^{-1}}=\bar{\xi}_{n}$.

\section{The group $\mathrm{GL}_{4}(q)$}

Here we determine explicitly the entries $\lambda_{y, y^{\prime}}$, of the representing matrix $S$ of the element $s=s_{3}$ for the group $\mathrm{GL}_{4}(q)$.

According to Theorem 10 we have to determine for each $y \in Y$ the Bruhat decomposition of the elements $y^{-1} s y^{\prime}, y^{\prime} \in Y$. Instead of considering these elements, we may equivalently consider for each $y \in Y$ the elements sya, $a \in X_{\psi} \backslash Z$, and determine whether such an element belongs to $A Z$ or not.

Lemma 12. Let $a=u t w \in X_{\psi} \backslash Z, w \in W, t \in T, u \in U_{w^{-1}}$ and $y=u^{\prime} t_{0} w^{\prime}, w^{\prime} \in W_{0}$, $t_{0} \in T_{0}, u^{\prime} \in U_{w^{\prime}-1}$. Then sya $\in A Z$ if and only if $s w^{\prime} w \in W_{0}$ and $u \in \prod X_{\alpha}$, $\alpha \in \Phi^{+} \cap w\left(\Phi_{0}^{-}\right)$

where $\Phi_{0}$ is the subsystem of $\Phi$ generated by the simple roots $\alpha_{i}, i=1, \ldots, n-2$.

Proof. We have $s y a=u^{\prime s} t_{0}^{s} s w^{\prime} w u^{w} t^{w}$. Since $w^{\prime} \in W_{0}$ and $u^{\prime} \in U_{w^{\prime}-1}=\prod_{\alpha \in \Phi^{+} \cap w^{\prime}\left(\Phi^{-}\right)} X_{\alpha}$, we see that $u^{\prime}$ is of the form

$$
\left(\begin{array}{ll}
B & O \\
O & 1
\end{array}\right)
$$

where $B$ is an upper unitriangular matrix of degree $n-1$. Thus $u^{\prime s} \in A$. Also $t_{0}^{s}, t^{w} \in A Z$. Suppose that $s y a \in A Z$; that is, $s w^{\prime} w u^{w} \in A Z$. Since $u \in U_{w^{-1}}, u^{w} \in{ }^{w_{0}} U \cap w^{-1} U=$ $\prod X_{\alpha}$; that is, $u^{w}$ is a lower unitriangular matrix. Now, the left multiplication $\alpha \in w^{-1}\left(\Phi^{+}\right) \cap \Phi^{-}$

action of $s w^{\prime} w$ on $u^{w}$ changes the rows of $u^{w}$. Since $s w^{\prime} w u^{w} \in A Z, s w^{\prime} w$ must leave the last row of $u^{w}$ fixed. Thus the last row of $s w^{\prime} w u^{w}$ must be that of $u^{w}$, and so $u^{w} \in$ $A \cap \prod_{\alpha \in w^{-1}\left(\Phi^{+}\right) \cap \Phi^{-}} X_{\alpha}=\prod_{\alpha \in w^{-1}\left(\Phi^{+}\right) \cap \Phi_{0}^{-}} X_{\alpha}$. Therefore $s w^{\prime} w \in W_{0}$ and $u \in \prod_{\alpha \in \Phi^{+} \cap w\left(\Phi_{0}^{-}\right)} X_{\alpha}$. The converse is obvious.

Thus for a given $w^{\prime} \in W_{0}$ we have to determine the elements $w \in N_{\psi} \cap W$ that satisfy the condition that $s w^{\prime} w \in W_{0}$. For this, we let $\sigma_{k}$ be the permutation matrix that corresponds 
to the permutation $(k, n-1), k=1, \ldots, n-1$. Then the set $\left\{\sigma_{k} \mid k=1, \ldots, n-1\right\}$ is a set of right coset representatives of the Weyl subgroup $W_{00}$ of $W_{0}$ which is generated by the reflections defined by the simple roots $\alpha_{1}, \ldots, \alpha_{n-3}$.

Corollary 13. Let $a$ and $y$ be the elements considered in Lemma 12. Suppose $w^{\prime} \in W_{00} \sigma_{k}$ for some $k=1, \ldots, n-1$. Then sya $\in A$ if and only if $t_{0}^{s} t^{w} \in T_{0}, w\left(e_{n}\right)=e_{k}$ and $u \in \quad \prod \quad X_{\alpha}$, where $\Phi_{k}^{-}=\varnothing$ if $k=1$; otherwise $\Phi_{k}^{-}$is the set of negative roots $\alpha \in \Phi^{+} \cap w\left(\Phi_{0}^{-} \backslash \Phi_{k}^{-}\right)$

of the subsystem $\Phi_{k}$ of $\Phi$ which is generated by the roots $\alpha_{n-1}, \ldots, \alpha_{n-(k-2)}, \alpha_{n-(k-1)}$.

Proof. Let $w^{\prime} \in W_{00} \sigma_{k}, k \in\{1, \ldots, n-1\}$. Suppose that sya $\in A$. Then by Lemma 12 we have $s w^{\prime} w \in W_{0}$ and $u \in \prod_{\alpha \in \Phi^{+} \cap w\left(\Phi_{0}^{-}\right)} X_{\alpha}$. Let $w\left(e_{n}\right)=e_{k^{\prime}}$. Then $k^{\prime} \neq n$, since the only element $w$ in $N_{\psi} \cap W$ that fixes $e_{n}$ is the identity, and in this case we should have $s w^{\prime} \in W_{0}$, which does not hold. Suppose that $k^{\prime} \neq k$. Then if $w^{\prime}=w^{\prime \prime} \sigma_{k}, w^{\prime \prime} \in W_{00}$, we have $s w^{\prime \prime} \sigma_{k} w\left(e_{n}\right)=s w^{\prime \prime} \sigma_{k}\left(e_{k^{\prime}}\right)=s w^{\prime}\left(e_{i}\right)$, where $i$ can be either $k$ (if $k^{\prime}=n-1$ ) or $k^{\prime}$ (if $k^{\prime} \neq n-1$ ) and so $i \in\{1, \ldots, n-2\}$. Thus $s w^{\prime \prime}\left(e_{i}\right)=e_{j}, j \in\{1,2, \ldots, n-2\}$. This means that $s w^{\prime} w \notin W_{0}$ as $s w^{\prime} w\left(e_{n}\right)=e_{j} \neq e_{n}$. Therefore we must have $w\left(e_{n}\right)=e_{k}$ and so, by Lemma 6, $w$ must be a permutation matrix of the form

$$
\left(\begin{array}{ccc}
O & & I_{k} \\
& . \cdot & \\
I_{v} & & O
\end{array}\right) \text {. }
$$

Thus, if $k>1$, we have $w\left(e_{n-\lambda}-e_{n-\lambda^{\prime}}\right)=e_{k-\lambda}-e_{k-\lambda^{\prime}}$ for $\lambda, \lambda^{\prime}, 0 \leqslant \lambda^{\prime}<\lambda \leqslant k-1$. This means that $w\left(\Phi_{k}^{+}\right) \subseteq \Phi^{+}$. Therefore we have $\Phi^{+} \cap w\left(\Phi_{0}^{-}\right)=\Phi^{+} \cap w\left(\Phi_{0}^{-} \backslash \Phi_{k}^{-}\right)$ as required. Finally, we notice that $s w^{\prime} w u^{w} \in A Z$ if and only if $s w^{\prime} w u^{w} \in A$, and so $t_{0}^{s} s w^{\prime} w u^{w}\left(t_{0}^{s}\right)^{-1} \in A$. Thus if $s y a \in A$ then $t_{0}^{s} t^{w} \in A \cap T=T_{0}$. The converse is obvious.

Now we return to the case $G=\operatorname{GL}_{4}(q)$. Here the root system is the set $\Phi=\left\{ \pm \alpha_{1}, \pm \alpha_{2}\right.$, $\left.\pm \alpha_{3}, \pm\left(\alpha_{1}+\alpha_{2}\right), \pm\left(\alpha_{2}+\alpha_{3}\right), \pm\left(\alpha_{1}+\alpha_{2}+\alpha_{3}\right)\right\}$. For the root subgroups $X_{\alpha_{i}}, i=1,2,3$, we shall write $X_{i}$, and $X_{12}, X_{23}, X_{123}$ for the subgroups $X_{\alpha_{1}+\alpha_{2}}, X_{\alpha_{2}+\alpha_{3}}, X_{\alpha_{1}+\alpha_{2}+\alpha_{3}}$, respectively. A typical element of the subgroup $X_{i}, i=1,2,3$, will be denoted by $x_{i}$ or $x_{i}^{\prime}$, etc., and similar notation will be used for the elements of the groups $X_{12}, X_{23}, X_{123}$.

Let $s, s_{1}$ and $s_{2}$ be the permutation matrices

$$
\left(\begin{array}{llll}
1 & 0 & 0 & 0 \\
0 & 1 & 0 & 0 \\
0 & 0 & 0 & 1 \\
0 & 0 & 1 & 0
\end{array}\right),\left(\begin{array}{llll}
0 & 1 & 0 & 0 \\
1 & 0 & 0 & 0 \\
0 & 0 & 1 & 0 \\
0 & 0 & 0 & 1
\end{array}\right) \text { and }\left(\begin{array}{llll}
1 & 0 & 0 & 0 \\
0 & 0 & 1 & 0 \\
0 & 1 & 0 & 0 \\
0 & 0 & 0 & 1
\end{array}\right)
$$

respectively.

We denote by $T_{i}, i=1,2,3,4,5,6$, the subgroups of $T$ that consist respectively of the 
diagonal matrices of the form

$$
\begin{aligned}
& \left(\begin{array}{llll}
\mu & & & \\
& v & & \\
& & v & \\
& & & v
\end{array}\right),\left(\begin{array}{llll}
\mu & & & \\
& \mu & & \\
& & \mu & \\
& & & v
\end{array}\right),\left(\begin{array}{llll}
\mu & & & \\
& v & & \\
& & \rho & \\
& & & \rho
\end{array}\right), \\
& \left(\begin{array}{llll}
\mu & & & \\
& \mu & & \\
& & v & \\
& & & \rho
\end{array}\right),\left(\begin{array}{llll}
\mu & & & \\
& v & & \\
& & v & \\
& & & \rho
\end{array}\right) \text { and }\left(\begin{array}{llll}
\mu & & & \\
& \mu & & \\
& & v & \\
& & & v
\end{array}\right) \text {. }
\end{aligned}
$$

By Lemma 6 the subset $N_{\psi}$, which consists of $q^{3}(q-1)$ monomial matrices, is the set $\left\{t_{i} w_{i} \mid t_{i} \in T_{i}, i=1, \ldots, 6\right\} \cup T w_{0} \cup Z$, where $w_{0}=s_{1} s_{2} s_{1} s s_{2} s_{1}, w_{1}=s_{1} s_{2} s, w_{2}=s s_{2} s_{1}$, $w_{3}=s_{1} s_{2} s_{1} s s_{2}, w_{4}=s_{2} s_{1} s s_{2} s_{1}, w_{5}=s_{1} s_{2} s_{2} s_{1}$ and $w_{6}=s_{2} s_{1} s s_{2}, Z$ being the center of $G$. Using Corollary 13 we consider the right coset representatives $\sigma_{k}$ of $W_{00}$ in $W_{0}$. We have $W_{0}=\left\{1, s_{1}, s_{2}, s_{1} s_{2}, s_{2} s_{1}, s_{1} s_{2} s_{1}\right\}, W_{00}=\left\{1, s_{1}\right\}$ and $\sigma_{1}=s_{1} s_{2} s_{1}, \sigma_{2}=s_{2}, \sigma_{3}=1$. The subgroups $U_{w^{-1}}, w \in W_{0}$, of $U$ are the following:

$$
\begin{aligned}
& U_{1^{-1}}=1, U_{s_{1}^{-1}}=X_{1}, U_{s_{2}^{-1}}=X_{2}, U_{\left(s_{1} s_{2}\right)^{-1}}=X_{1} X_{12}, U_{\left(s_{2} s_{1}\right)^{-1}}=X_{2} X_{12} \text { and } \\
& U_{\left(s_{1} s_{2} s_{1}\right)^{-1}}=X_{1} X_{2} X_{12} .
\end{aligned}
$$

Thus the set $Y$ of the left coset representatives of the subgroup $U$ in the affine subgroup $A$ of $G$ is

$$
\begin{aligned}
Y= & \left\{t_{0}, x_{1} t_{0} s_{1}, x_{2} t_{0} s_{2}, x_{1} x_{12} t_{0} s_{1} s_{2}, x_{2} x_{12} t_{0} s_{2} s_{1}, x_{1} x_{2} x_{12} t_{0} s_{1} s_{2} s_{1} \mid\right. \\
& \left.t_{0} \in T_{0}, x_{1} \in X_{1}, x_{2} \in X_{2}, x_{12} \in X_{12}\right\} .
\end{aligned}
$$

Now we classify the elements of $Y$ into three classes according to the right coset representatives $\sigma_{1}, \sigma_{2}$ and $\sigma_{3}$.

$\sigma_{1}$ gives rise to the class

$$
\Sigma_{1}=\left\{x_{1} x_{2} x_{12} t_{0} s_{1} s_{2} s_{1}, x_{2} x_{12} t_{0} s_{2} s_{1} \mid x_{1} \in X_{1}, x_{2} \in X_{2}, x_{12} \in X_{12}, t_{0} \in T_{0}\right\},
$$

$\sigma_{2}$ gives rise to the class

$$
\Sigma_{2}=\left\{x_{2} t_{0} s_{2}, x_{1} x_{12} t_{0} s_{1} s_{2} \mid x_{1} \in X_{1}, x_{2} \in X_{2}, x_{12} \in X_{12}, t_{0} \in T_{0}\right\}, \text { and }
$$

$\sigma_{3}$ gives rise to the class

$$
\Sigma_{3}=\left\{t_{0}, x_{1} t_{0} s_{1} \mid x_{1} \in X_{1}, t_{0} \in T_{0}\right\} .
$$

Let $t_{0}=\operatorname{diag}\left(\kappa_{1}, \kappa_{2}, \kappa_{3}, 1\right)$ be an element of $T_{0}$. Then by simple calculations it turns out that if $y \in \Sigma_{1}$ then the elements $a \in X_{\psi} \backslash Z$ satisfying the condition sya $\in A$ are of the form $t_{1} w_{1}, x_{2}^{\prime \prime} x_{23}^{\prime \prime} t_{3} w_{3}, x_{3}^{\prime \prime} x_{23}^{\prime \prime} t_{5} w_{5}$ and $x_{2}^{\prime \prime} x_{3}^{\prime \prime} x_{23}^{\prime \prime} t w_{0}$, where $t_{1}=\operatorname{diag}\left(\kappa_{3}^{-1}, v, v, v\right) \in T_{1}$, $t_{3}=\operatorname{diag}\left(\kappa_{3}^{-1}, v, \rho, \rho\right) \in T_{3}, t_{5}=\operatorname{diag}\left(\kappa_{3}^{-1}, v, \nu, \rho\right) \in T_{5}, t=\operatorname{diag}\left(\kappa_{3}^{-1}, \nu, \rho, \tau\right) \in T$, $x_{2}^{\prime \prime} \in X_{2}, x_{3}^{\prime \prime} \in X_{3}$ and $x_{23}^{\prime \prime} \in X_{23}$. If $y \in \Sigma_{2}$, then the corresponding $a \in X_{\psi} \backslash Z$ are of the form $x_{3}^{\prime \prime} x_{12}^{\prime \prime} x_{123}^{\prime \prime} t_{4} w_{4}$ or of the form $x_{12}^{\prime \prime} x_{123}^{\prime \prime} t_{6} w_{6}$, where $t_{4}=\operatorname{diag}\left(\kappa_{3}^{-1}, \kappa_{3}^{-1}, v, \rho\right) \in T_{4}$, $t_{6}=\operatorname{diag}\left(\kappa_{3}^{-1}, \kappa_{3}^{-1}, v, v\right) \in T_{6}, x_{3}^{\prime \prime} \in X_{3}, x_{12}^{\prime \prime} \in X_{12}$ and $x_{123}^{\prime \prime} \in X_{123}$. Finally for the 
elements of $\Sigma_{3}$ the corresponding $a \in X_{\psi} \backslash Z$ are elements of the form $x_{23}^{\prime \prime} x_{123}^{\prime \prime} t_{2} w_{2}$, where $t_{2}=\operatorname{diag}\left(\kappa_{3}^{-1}, \kappa_{3}^{-1}, \kappa_{3}^{-1}, v\right) \in T_{2}, x_{23}^{\prime \prime} \in X_{23}$ and $x_{123}^{\prime \prime} \in X_{123}$.

To describe the Bruhat decomposition of $s y a$ we put $y_{1}=t_{0}, y_{2}=x_{1} t_{0} s_{1}, y_{3}=x_{2} t_{0} s_{2}$, $y_{4}=x_{1} x_{12} t_{0} s_{1} s_{2}, y_{5}=x_{2} x_{12} t_{0} s_{2} s_{1}, y_{6}=x_{1} x_{2} x_{12} t_{0} s_{1} s_{2} s_{1}$ and $a_{0}=x_{2}^{\prime \prime} x_{3}^{\prime \prime} x_{23}^{\prime \prime} t w_{0}, a_{1}=$ $t_{1} w_{1}, a_{2}=x_{23}^{\prime \prime} x_{123}^{\prime \prime} t_{2} w_{2}, a_{3}=x_{2}^{\prime \prime} x_{23}^{\prime \prime} t_{3} w_{3}, a_{4}=x_{3}^{\prime \prime} x_{12}^{\prime \prime} x_{123}^{\prime \prime} t_{4} w_{4}, a_{5}=x_{3}^{\prime \prime} x_{23}^{\prime \prime} t_{5} w_{5}$ and $a_{6}=x_{12}^{\prime \prime} x_{123}^{\prime \prime} t_{6} w_{6}$. For $i=1, \ldots, 6$ and $j=0,1, \ldots, 6$ let $y_{i j}^{\prime}$ be the element of $Y$ and $v_{i j} \in U$ such that $y_{i j}^{\prime} v_{i j}$ is the Bruhat decomposition of $s y_{i} a_{j}$.

Tables 1 and 2 give all possible $y_{i j}^{\prime}$ and $v_{i j}$ such that $s y_{i} a_{j} \in A$. In these tables we have put

$$
\begin{aligned}
& d_{1}=\left(t_{0} t_{2}\right)^{s}, d_{2}=\left(t_{0} t_{1}^{s_{1}}\right)^{s}, d_{3}=\left(t_{0} t_{6}^{s_{2}}\right)^{s}, d_{4}=\left(t_{0} t_{4}^{s_{2}}\right)^{s}, d_{5}=\left(t_{0} t_{6}^{s_{2} s_{1}}\right)^{s}, \\
& d_{6}=\left(t_{0} t_{4}^{s_{2} s_{1}}\right)^{s}, d_{7}=\left(t_{0} t_{1}^{s_{1} s_{2}}\right)^{s}, d_{8}=\left(t_{0} t_{5}^{s_{1} s_{2}}\right)^{s}, d_{9}=\left(t_{0} t_{3}^{s_{1} s_{2}}\right)^{s}, d_{10}=\left(t_{0} t^{s_{1} s_{2}}\right)^{s}, \\
& d_{11}=\left(t_{0} t_{1}^{s_{1} s_{2} s_{1}}\right)^{s}, d_{12}=\left(t_{0} t_{5}^{s_{1} s_{2} s_{1}}\right)^{s}, d_{13}=\left(t_{0} t_{3}^{s_{1} s_{2} s_{1}}\right)^{s}, d_{14}=\left(t_{0} t^{s_{1} s_{2} s_{1}}\right)^{s} .
\end{aligned}
$$

Moreover, the elements $\beta_{1}, \gamma_{1}, d$ and $f$ appearing in these tables are determined as follows. For the element $x_{12}^{\prime \prime} \neq 1$ in $X_{12}$ in the expression of $a_{4}$ and $a_{6}$ we have $x_{12}^{1 \prime s s_{2} s_{1} t_{0}^{-1} s} \in X_{-1}$, where $X_{-1}$ denotes the subgroup $X_{-a_{1}}$. As one can see, the Bruhat decomposition of the non-trivial elements in $X_{-1}$ has the form $\beta_{1} d s_{1} \beta_{1}$, where $\beta_{1} \in X_{1}, d \in T$. Also, for the element $x_{2}^{\prime \prime} \neq 1$ of $X_{2}$ in the expression of $a_{0}$ and $a_{3}$, we have $x_{2}{ }^{1 / s_{1} s_{2} s_{1} t_{0}^{-1} s} \in X_{-1}$, and so its Bruhat decomposition should be of the form $\gamma_{1} f s_{1} \gamma_{1}, \gamma_{1} \in X_{1}, f \in T$. $\mathrm{m}$, In Table 1 we have written $y_{i j}^{\prime}=x_{1}^{\prime} x_{2}^{\prime} x_{12}^{\prime} t_{0}^{\prime} w^{\prime}$, where $x_{1}^{\prime} \in X_{1}, x_{2}^{\prime} \in X_{2}, x_{12}^{\prime} \in X_{12}, t_{0}^{\prime} \in T_{0}$ and $w^{\prime} \in W_{0}$. Thus, for example, if $i=3, j=6$, we have $x_{1}^{\prime}=x_{12}^{\prime \prime s s_{2} t_{0}^{-1}}, x_{2}^{\prime}=1, x_{12}^{\prime}=x_{123}^{\prime \prime s 2} t_{0}^{-1} s$, $t_{0}^{\prime}=d_{3}=\left(t_{0} t_{6}^{s_{2}}\right)^{s}$ and $w^{\prime}=s_{1} s_{2}$.

Now, using Tables 1 and 2, we can determine all pairs $\left(y, y^{\prime}\right) \in Y \times Y$ such that the Bruhat decompositions of the elements $y^{-1} s y^{\prime}$ are of the form $a v$, where $a$ is one of the elements in $X_{\psi} \backslash Z$ and $v \in U$.

By Theorem 10 the entry $\lambda_{y, y^{\prime}}$ of the matrix $S$ corresponding to the pair $\left(y, y^{\prime}\right)$ is equal to $\xi_{a} \psi\left(v^{-1}\right)$. Below, we give these entries in terms of $y$ and $y^{\prime}$. For example, let us take $y=t_{0}$ and $a=a_{2}=x_{23}^{\prime \prime} x_{123}^{\prime \prime} t_{2} s s_{2} s_{1}$ where $x_{23}^{\prime \prime} \in X_{23}, x_{123}^{\prime \prime} \in X_{123}$ and $t_{2} \in T_{2}$. The Bruhat decomposition of $s y a$ is $s y a=y^{\prime} v$, where $y^{\prime}=x_{2}^{\prime} x_{12}^{\prime} t_{0}^{\prime} s_{2} s_{1}, x_{2}^{\prime}=x_{23}^{\prime \prime \prime} t^{-1} s, x_{12}^{\prime}=x_{123}^{\prime \prime t_{0}^{-1} s}$, $t_{0}^{\prime}=d_{1}=\left(t_{0} t_{2}\right)^{s}$ and $v=1$ by Tables 1 and 2 .

Thus we have $\lambda_{y, y^{\prime}}=\xi_{x_{23}^{\prime \prime} x_{123}^{\prime \prime} t_{2} s s_{2} s_{1}}=\xi_{t_{2} s s_{2} s_{1}}(*), \quad$ as $\psi\left(x_{23}^{\prime \prime-1} x_{123}^{\prime \prime-1}\right)=1$. Eliminating $t_{2}$ in (*) we get $\lambda_{y, y^{\prime}}=\xi_{t_{0}^{-1} s t_{0}^{\prime} s_{2} s_{1}}$ if $t_{0}^{-1} t_{0}^{\prime s} \in T_{2}$, otherwise $\lambda_{y, y^{\prime}}=0$. Thus the function that gives the entry $\lambda_{y, y^{\prime}}$ where $y=t_{0}$ and $y^{\prime}=x_{2}^{\prime} x_{12}^{\prime} t_{0}^{\prime} s_{2} s_{1}$ is of the form

$$
\Xi_{\left(t_{0} ; t_{0}^{\prime}\right)}=\left\{\begin{array}{l}
\xi_{t_{0}^{-1} s t_{0}^{\prime} s_{2} s_{1}} \text { if } t_{0}^{-1} t_{0}^{\prime s} \in T_{2} \\
0 \text { otherwise. }
\end{array}\right.
$$


The Cuspidal Modules of the Finite General Linear Groups

Table 1: The form of elements $a$.

\begin{tabular}{|c|c|c|c|c|c|}
\hline $\mathbf{y}_{\mathbf{i j}}$ & $\mathbf{x}_{1}$ & $\mathbf{x}_{2}$ & $\mathbf{x}_{12}^{\prime}$ & $\mathbf{t}_{\mathbf{0}}^{\prime}$ & $\mathbf{w}^{\prime}$ \\
\hline$y_{12}^{\prime}$ & & $x_{23}^{\prime \prime t_{0}^{-1} s}$ & $x_{123}^{\prime \prime t_{0}^{-1} s}$ & $d_{1}$ & $s_{2} s_{1}$ \\
\hline & $x_{1}$ & $x_{123}^{\prime \prime s_{1} t_{0}^{-1} s}$ & $x_{23}^{\prime \prime s_{1} t_{0}^{-1} s}$ & $d_{2}$ & $s_{1} s_{2} s_{1}$ \\
\hline$y_{36}^{\prime}$ & $x_{12}^{1 / S_{2} t_{0}^{-1}}$ & & $x_{123}^{1 / s_{2} t_{0}^{-1} s}$ & $d_{3}$ & $s_{1} s_{2}$ \\
\hline$y_{34}^{\prime}$ & $x_{12}^{1 / s_{2} t_{0}^{-1}}$ & $x_{3}{ }^{\prime \prime s_{2} t_{0}^{-1} s}$ & $\left(\left[x_{3}^{\prime \prime-1}, x_{12}^{\prime \prime-1}\right] x_{123}^{\prime \prime}\right)^{s_{2} t_{0}^{-1} s}$ & $d_{4}$ & $s_{1} s_{2} s_{1}$ \\
\hline $\begin{array}{c}y_{46}^{\prime} \\
\left(x_{12}^{\prime \prime}=1\right)\end{array}$ & & $x_{123}^{\prime \prime s_{2} s_{1} t_{0}^{-1} s}$ & & $d_{5}$ & $s_{2}$ \\
\hline $\begin{array}{c}y_{46}^{\prime} \\
\left(x_{12}^{\prime \prime} \neq 1\right)\end{array}$ & $x_{1} \beta_{1}$ & & {$\left[\left(x_{123}^{\prime \prime s_{2} s_{1} t_{0}^{-1} s}\right)^{-1}, \beta_{1}^{-1}\right]$} & $d d_{5}^{s_{1}}$ & $s_{1} s_{2}$ \\
\hline $\begin{array}{c}y_{44}^{\prime} \\
\left(x_{12}^{\prime \prime}=1\right)\end{array}$ & & $x_{123}^{\prime \prime s_{2} s_{1} t_{0}^{-1} s}$ & $x_{3}{ }^{\prime \prime s_{2} s_{1} t_{0}^{-1} s}\left[x_{1}^{-1},\left(x_{123}^{\prime \prime s_{2} s_{1} t_{0}^{-1} s}\right)^{-1}\right]$ & $d_{6}$ & $s_{2} s_{1}$ \\
\hline $\begin{array}{c}y_{44}^{\prime} \\
\left(x_{12}^{\prime \prime} \neq 1\right)\end{array}$ & $x_{1} \beta_{1}$ & $x_{123}^{\prime \prime s_{2} s_{1} t_{0}^{-1} s}$ & {$\left[\left(x_{123}^{\prime \prime s_{2} s_{1} t_{0}^{-1} s}\right)^{-1}, \beta_{1}^{-1}\right] x_{3}{ }^{\prime \prime s_{2} s_{1} t_{0}^{-1} s}$} & $d d_{6}^{s_{1}}$ & $s_{1} s_{2} s_{1}$ \\
\hline$y_{51}^{\prime}$ & & & & $d_{7}$ & 1 \\
\hline$y_{55}^{\prime}$ & & $x_{3}^{1 / 1 S_{2} t_{0}^{-1} s}$ & $x_{23}^{1 / S_{1} s_{2} t_{0}^{-1} s}$ & $d_{8}$ & $s_{2} s_{1}$ \\
\hline$y_{53}^{\prime}$ & $\begin{array}{l}x_{2}^{\prime \prime S} s_{1} s_{2} t_{0}^{-1} \\
{ }^{\prime \prime S 1} s_{2} t_{0}^{-1}\end{array}$ & & $x_{23}^{\prime \prime s_{1} s_{2} t_{0}^{-1} s}$ & $d_{9}$ & $s_{1} s_{2}$ \\
\hline$y_{50}^{\prime}$ & $x_{2}^{\prime \prime s S_{1} s_{2} t_{0}^{-1}}$ & $x_{3}^{1 / s_{2} t_{0}^{-1} s}$ & $x_{23}^{\prime \prime s} s_{1} s_{2} t_{0}^{-1} s$ & $d_{10}$ & $s_{1} s_{2} s_{1}$ \\
\hline$y_{61}^{\prime}$ & $x_{1}$ & & & $d_{11}$ & $s_{1}$ \\
\hline$y_{65}^{\prime}$ & $x_{1}$ & $x_{23}^{\prime \prime s_{1} s_{2} s_{1} t_{0}^{-1} s}$ & $x_{3}^{1 / s_{2} s_{1} t_{0}^{-1} s}$ & $d_{12}$ & $s_{1} s_{2} s_{1}$ \\
\hline $\begin{array}{c}y_{63}^{\prime} \\
\left(x_{2}^{\prime \prime}=1\right)\end{array}$ & & $x_{23}^{\prime \prime s_{1} s_{2} s_{1} t_{0}^{-1} s}$ & & $d_{13}$ & $s_{2}$ \\
\hline $\begin{array}{c}y_{63}^{\prime} \\
\left(x_{2}^{\prime \prime} \neq 1\right)\end{array}$ & $x_{1} \gamma_{1}$ & & {$\left[\left(x_{23}^{\prime \prime s_{1} s_{2} s_{1} t_{0}^{-1} s}\right)^{-1}, \gamma_{1}^{-1}\right]$} & $f d_{13}^{s_{1}}$ & $s_{1} s_{2}$ \\
\hline $\begin{array}{c}y_{60}^{\prime} \\
\left(x_{2}^{\prime \prime}=1\right)\end{array}$ & & $x_{23}^{\prime \prime s_{1} s_{2} s_{1} t_{0}^{-1} s}$ & {$\left[x_{1}^{-1},\left(x_{23}{ }^{\prime \prime S_{1} s_{2} s_{1} t_{0}^{-1} s}\right)^{-1}\right] x_{3}{ }^{1 / S_{2} s_{1} t_{0}^{-1} s}$} & $d_{14}$ & $s_{2} s_{1}$ \\
\hline $\begin{array}{c}y_{60} \\
\left(x_{2}^{\prime \prime} \neq 1\right)\end{array}$ & $x_{1} \gamma_{1}$ & $\left(\left[x_{2}^{\prime \prime-1}, x_{3}^{\prime \prime-1}\right]\right.$ & {$\left[\left(\left(\left[x_{2}^{\prime \prime-1}, x_{3}^{\prime \prime-1}\right] x_{23}^{\prime \prime}\right)^{s_{1} s_{2} s_{1} t_{0}^{-1} s}\right)^{-1}\right.$} & $f d_{14}^{s_{1}}$ & $s_{1} s_{2} s_{1}$ \\
\hline & & $\left.x_{23}^{\prime \prime}\right)^{s_{1} s_{2} s_{1} t_{0}^{-1} s}$ & $\left.\gamma_{1}^{-1}\right] x_{3}^{1 / s_{2} s_{1} t_{0}^{-1} s}$ & & \\
\hline
\end{tabular}


Table 2: The form of the elements $v$.

$$
\begin{aligned}
& v_{12}=1 \\
& v_{22}=1 \\
& v_{36}=x_{2}^{s d_{3} s_{1} s_{2}}\left[x_{2}^{-1},\left(x_{12}^{\prime \prime s_{2} t_{0}^{-1}}\right)^{-1}\right]^{s d_{3} s_{1} s_{2}} \in X_{123} X_{3} \\
& v_{34}=x_{2}^{s d_{4} s_{1} s_{2} s_{1}}\left[x_{2}^{-1},\left(x_{12}^{\prime \prime s_{2} t_{0}^{-1}}\right)^{-1}\right]^{s d_{4} s_{1} s_{2} s_{1}} \in X_{23} X_{3} \\
& v_{46}=x_{1}^{d_{5} s_{2}}\left[x_{1}^{-1},\left(x_{123}^{\prime \prime s_{2} s_{1} t_{0}^{-1}}\right)^{-1}\right]^{s d_{5} s_{2}} x_{12}^{s d_{5} s_{2}} \in X_{12} X_{1} X_{123} \text {, if } x_{12}^{\prime \prime}=1 \\
& v_{46}=x_{123}^{1 / s_{2} s_{1} t_{0}^{-1} s d s_{1} d_{5} s_{2}} x_{12}^{s d s_{1} d_{5} s_{2}} \beta_{1}^{d_{5} s_{2}} \in X_{1} X_{3} X_{12} \text {, if } x_{12}^{\prime \prime} \neq 1 \\
& v_{44}=x_{1}^{d_{6} s_{2} s_{1}} x_{12}^{s d_{6} s_{2} s_{1}} \in X_{2} X_{23} \text {, if } x_{12}^{\prime \prime}=1 \\
& v_{44}=x_{12}^{s d s_{1} d_{6} s_{2}} \beta_{1}^{d_{6} s_{2} s_{1}} \in X_{3} X_{2} \text {, if } x_{12}^{\prime \prime} \neq 1 \\
& v_{51}=x_{2}^{s d_{7}} x_{12}^{s d_{7}} \in X_{23} X_{123} \\
& v_{55}=x_{2}^{s d_{8} s_{2}} x_{12}^{s d_{8} s_{2} s_{1}} \in X_{3} X_{23} \\
& v_{53}=x_{2}^{s d_{9} s_{1} s_{2}}\left(\left[x_{2}^{-1},\left(x_{2}^{\prime \prime s_{1} s_{2} t_{0}^{-1}}\right)^{-1}\right] x_{12}\right)^{s d_{9} s_{1} s_{2}} \in X_{123} X_{3} \\
& v_{50}=x_{2}^{s d_{10} s_{1} s_{2} s_{1}}\left(\left[x_{2}^{-1},\left(x_{2}^{\prime \prime s_{1} s_{2} t_{0}^{-1}}\right)^{-1}\right] x_{12}\right)^{s d_{10} s_{1} s_{2}} \in X_{23} X_{3} \\
& v_{61}=x_{2}^{s d_{11} s_{1}} x_{12}^{s d_{11} s_{1}} \in X_{123} X_{23} \\
& v_{65}=x_{2}^{s d_{12} s_{1} s_{2} s_{1}} x_{12}^{s d_{12} s_{1} s_{2}} \in X_{23} X_{3} \\
& v_{63}=x_{1}^{d_{13} s_{2}}\left[x_{1}^{-1},\left(x_{23}^{\prime \prime s_{1} s_{2} s_{1} t_{0}^{-1}}\right)^{-1}\right]^{s d_{13} s_{2}} x_{2}^{s d_{13} s_{2}} x_{12}^{s d_{13} s_{2}} \in X_{12} X_{1} X_{3} X_{123} \text { if } x_{2}^{\prime \prime}=1 \\
& v_{63}=x_{2}^{s f s_{1} d_{13} s_{2}}\left(\left[x_{2}^{-1}, \gamma_{1}^{-1}\right] x_{12}\right)^{s f s_{1} d_{13} s_{2}} x_{23}^{1 / s_{1} s_{2} s_{1} t_{0}^{-1} s f s_{1} d_{13} s_{2}} \gamma_{1}^{d_{13} s_{2}} \in X_{123} X_{3} X_{1} X_{12} \\
& \text { if } x_{2}^{\prime \prime} \neq 1 \\
& v_{60}=x_{1}^{d_{14 s_{2} s_{1}}} x_{2}^{s d_{14 s_{2}}} x_{12}^{s d_{14} s_{2} s_{1}} \in X_{2} X_{3} X_{23} \\
& \text { if } x_{2}^{\prime \prime}=1 \\
& v_{60}=x_{2}^{s f s_{1} d_{14} s_{2} s_{1}}\left(\left[x_{2}^{-1}, \gamma_{1}^{-1}\right] x_{12}\right)^{s f s_{1} d_{14} s_{2}} \gamma_{1}^{d_{14} s_{2} s_{1}} \in X_{23} X_{3} X_{2} \text { if } x_{2}^{\prime \prime} \neq 1
\end{aligned}
$$


Our calculations have shown that the matrix $S$ with respect to the ordered basis $\left\{t_{0} b\right.$, $x_{1} t_{0} s_{1} b, x_{2} t_{0} s_{2} b, x_{1} x_{12} t_{0} s_{1} s_{2} b, x_{2} x_{12} t_{0} s_{2} s_{1} b, x_{1} x_{2} x_{12} t_{0} s_{1} s_{2} s_{1} b ; t_{0} \in T_{0}, x_{1} \in X_{1}, x_{2} \in$ $\left.X_{2}, x_{12} \in X_{12}\right\}$ is

$$
\left.\begin{array}{cccc}
0 & 0 & 0 & 0 \\
0 & 0 & 0 & 0 \\
0 & 0 & 0 & \Xi_{\left(t_{0}, x_{2} ; t_{0}^{\prime}, x_{1}^{\prime}\right)} \\
0 & 0 & \Xi_{\left(t_{0}, x_{1} ; t_{0}^{\prime}, x_{2}^{\prime}\right)} & \Xi_{\left(t_{0}, x_{1}, x_{12} ; t_{0}^{\prime}, x_{1}^{\prime}, x_{12}^{\prime}\right)} \\
\Xi_{\left(t_{0} ; t_{0}^{\prime}\right)}^{\prime \prime} & 0 & 0 & \Xi_{\left(t_{0}, x_{2}, x_{12} ; t_{0}^{\prime}, x_{1}^{\prime}\right)} \\
0 & \delta_{x_{1}, x_{1}^{\prime}}^{\prime} \Xi_{\left(t_{0} ; t_{0}^{\prime}\right)}^{\prime \prime \prime} & \Xi_{\left(t_{0}, x_{1}, x_{2} ; t_{0}^{\prime}, x_{2}^{\prime}\right)} & \Xi_{\left(t_{0}, x_{1}, x_{2}, x_{12} ; t_{0}^{\prime}, x_{1}^{\prime}, x_{12}^{\prime}\right)} \\
& \Xi_{\left(t_{0} ; t_{0}^{\prime}\right)} & \delta_{x_{1}, x_{1}^{\prime}} & \Xi_{\left(t_{0} ; t_{0}^{\prime}\right)}^{\prime} \\
& \Xi_{\left(t_{0}, x_{1} ; t_{0}^{\prime}, x_{2}^{\prime}, x_{12}^{\prime}\right)} & \Xi_{\left(t_{0}, x_{1}, x_{12} ; t_{0}^{\prime}, x_{1}^{\prime}, x_{2}^{\prime}, x_{12}^{\prime}\right)} \\
& \Xi_{\left(t_{0}, x_{2} ; t_{0}^{\prime}, x_{2}^{\prime}\right)} & \Xi_{\left(t_{0}, x_{2}, x_{12} ; t_{0}^{\prime}, x_{1}^{\prime}, x_{2}^{\prime}\right)} & \Xi_{\left(t_{0}, x_{1}, x_{2}, x_{12} ; t_{0}^{\prime}, x_{1}^{\prime}, x_{2}^{\prime}, x_{12}^{\prime}\right)}
\end{array}\right)
$$

where

$\Xi_{\left(t_{0} ; t_{0}^{\prime}\right)}=\left\{\begin{array}{l}\xi_{t_{0}^{-1} s t_{0}^{\prime} s_{2} s_{1}} \text { if } t_{0}^{-1} t_{0}^{\prime s} \in T_{2} \\ 0 \text { otherwise. }\end{array}\right.$

$\Xi_{\left(t_{0} ; t_{0}^{\prime}\right)}^{\prime}=\left\{\begin{array}{l}\xi_{s_{1} t_{0}^{-1} s t_{0}^{\prime} s_{1} s_{2} s_{1}} \text { if }\left(t_{0}^{-1} t_{0}^{\prime s}\right)^{s_{1}} \in T_{2} \\ 0 \text { otherwise. }\end{array}\right.$

$\Xi_{\left(t_{0}, x_{2} ; t_{0}^{\prime}, x_{1}^{\prime}\right)}=\left\{\begin{array}{l}\xi_{s_{2} t_{0}^{-1} s t_{0}^{\prime} s_{1} s_{2}} \psi\left(\left[x_{2}^{-1}, x_{1}^{\prime-1}\right]^{s t_{0}^{\prime} s_{1} s_{2}}\right) \text { if }\left(t_{0}^{-1} t_{0}^{s}\right)^{s_{2}} \in T_{6} \\ 0 \text { otherwise. }\end{array}\right.$

$\Xi_{\left(t_{0}, x_{2} ; t_{0}^{\prime}, x_{1}^{\prime}, x_{2}^{\prime}\right)}=\left\{\begin{array}{l}\xi_{s_{2} t_{0}^{-1} s t_{0}^{\prime} s_{1} s_{2} s_{1}} \psi\left(\left(x_{2}^{\prime-1}\right)^{s t_{0} s_{2}}\left[x_{2}^{-1}, x_{1}^{\prime-1}\right]^{s t_{0}^{\prime} s_{1} s_{2}}\right) \text { if }\left(t_{0}^{-1} t_{0}^{\prime s}\right)^{s_{2}} \in T_{4} \\ 0 \text { otherwise. }\end{array}\right.$

$\Xi_{\left(t_{0}, x_{1} ; t_{0}^{\prime}, x_{2}^{\prime}\right)}=\left\{\begin{array}{l}\xi_{s_{2} s_{1} t_{0}^{-1} s t_{0}^{\prime} s_{2}} \psi\left(\left[x_{1}^{-1}, x_{2}^{\prime-1}\right]^{t_{0}^{\prime} s_{2}}\right) \text { if }\left(t_{0}^{-1} t_{0}^{\prime s}\right)^{s_{1} s_{2}} \in T_{6} \\ 0 \text { otherwise. }\end{array}\right.$ 
$\Xi_{\left(t_{0}, x_{1}, x_{12} ; t_{0}^{\prime}, x_{1}^{\prime}, x_{12}^{\prime}\right)}=\left\{\begin{array}{l}\xi_{s_{2} s_{1} t_{0}^{-1} s_{1} s d^{-1} t_{0}^{\prime} s_{1} s_{2}} \psi\left(\left(\omega_{2} x_{12}^{s}\right)^{t_{0}^{\prime} s_{1} s_{2}}\right) \text { if } x_{1}^{\prime} \neq x_{1} \\ \text { and }\left(t_{0}^{-1}\right)^{s_{1} s_{2}}\left(d^{-1} t_{0}^{\prime}\right)^{s s_{2}} \in T_{6}, \text { where } d \in T \text { such that } \\ \left(x_{1}^{-1} x_{1}^{\prime}\right) d s_{1}\left(x_{1}^{-1} x_{1}^{\prime}\right) \in X_{-1} \backslash\{1\} \text { and } \\ \omega_{2} \in X_{2} \text { such that }\left[\omega_{2}^{-1}, x_{1} x_{1}^{\prime-1}\right]=x_{12}^{\prime} ; \\ 0 \text { otherwise. }\end{array}\right.$

$\Xi_{\left(t_{0}, x_{1} ; t_{0}^{\prime}, x_{2}^{\prime}, x_{12}^{\prime}\right)}=\left\{\begin{array}{l}\xi_{s_{2} s_{1} t_{0}^{-1} s t_{0}^{\prime} s_{2} s_{1}} \psi\left(\left(\left[x_{1}^{-1}, x_{2}^{\prime-1}\right] x_{12}^{\prime-1}\right)^{s t_{0} s_{1} s_{2}} x_{1}^{t_{0}^{\prime} s_{2} s_{1}}\right) \text { if }\left(t_{0}^{-1} t_{0}^{\prime s}\right)^{s_{1} s_{2}} \in T_{4} \\ 0 \text { otherwise. }\end{array}\right.$

$\Xi_{\left(t_{0}, x_{1}, x_{12} ; t_{0}^{\prime}, x_{1}^{\prime}, x_{2}^{\prime}, x_{12}^{\prime}\right)}=\left\{\begin{array}{l}\xi_{s_{2} s_{1} t_{0}^{-1} s_{1} s d^{-1} t_{0}^{\prime} s_{1} s_{2} s_{1}} \psi\left(\left(x_{12}^{\prime-1}\left[x_{2}^{\prime-1}, x_{1}^{\prime-1} x_{1}\right]\right)^{s t_{0} s_{1} s_{2}}\right. \\ \left.x_{12}^{s t_{0}^{\prime} s_{1} s_{2}}\left(x_{1}^{-1} x_{1}^{\prime}\right)^{s_{1} d^{-1}} t_{0}^{\prime} s_{1} s_{2} s_{1}\right) \\ \text { if } x_{1}^{\prime} \neq x_{1} \text { and }\left(t_{0}^{-1}\right)^{s_{1} s_{2}}\left(d^{-1} t_{0}^{\prime}\right)^{s s_{2}} \in T_{4} \\ \text { where } d \in T \text { such that }\left(x_{1}^{-1} x_{1}^{\prime}\right) d s_{1}\left(x_{1}^{-1} x_{1}^{\prime}\right) \in X_{-1} \backslash\{1\} \\ 0 \text { otherwise. }\end{array}\right.$

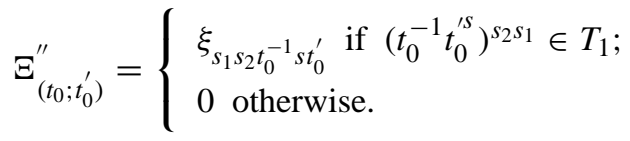

$\Xi_{\left(t_{0}, x_{2} ; t_{0}^{\prime}, x_{2}^{\prime}\right)}=\left\{\begin{array}{l}\xi_{s_{1} s_{2} t_{0}^{-1} s t_{0}^{\prime} s_{2} s_{1}} \psi\left(\left(x_{2}^{\prime-1}\right)^{s t_{0} s_{2}} x_{2}^{s t_{0}^{\prime} s_{2}}\right) \text { if }\left(t_{0}^{-1} t_{0}^{\prime s}\right)^{s_{2} s_{1}} \in T_{5} \\ 0 \text { otherwise. }\end{array}\right.$

$\Xi_{\left(t_{0}, x_{2}, x_{12} ; t_{0}^{\prime}, x_{1}^{\prime}\right)}=\left\{\begin{array}{l}\xi_{s_{1} s_{2} t_{0}^{-1} s t_{0}^{\prime} s_{1} s_{2}} \psi\left(\left(x_{1}^{\prime-1}\right)^{t_{0} s_{2} s_{1}}\left(\left[x_{2}^{-1}, x_{1}^{\prime-1}\right] x_{12}\right)^{s t_{0}^{\prime} s_{1} s_{2}}\right) \\ \text { if }\left(t_{0}^{-1} t_{0}^{\prime s}\right)^{s_{2} s_{1}} \in T_{3} ; \\ 0 \text { otherwise. }\end{array}\right.$

$\Xi_{\left(t_{0}, x_{2}, x_{12} ; t_{0}^{\prime}, x_{1}^{\prime}, x_{2}^{\prime}\right)}=\xi_{s_{1} s_{2} t_{0}^{-1} s t_{0}^{\prime} s_{1} s_{2} s_{1}} \psi\left(\left(x_{1}^{\prime-1} x_{2}^{\prime-1}\right)^{s t_{0} s_{2} s_{1}}\left(\left[x_{2}^{-1}, x_{1}^{\prime-1}\right] x_{12}\right)^{s t_{0}^{\prime} s_{1} s_{2}}\right)$. 
The Cuspidal Modules of the Finite General Linear Groups

$\Xi_{\left(t_{0} ; t_{0}^{\prime}\right)}^{\prime \prime \prime}=\left\{\begin{array}{l}\xi_{s_{1} s_{2} s_{1} t_{0}^{-1} s t_{0}^{\prime} s_{1}} \text { if }\left(t_{0}^{-1} t_{0}^{\prime s}\right)^{s_{1} s_{2} s_{1}} \in T_{1} \\ 0 \text { otherwise. }\end{array}\right.$

$\Xi_{\left(t_{0}, x_{1}, x_{2} ; t_{0}^{\prime}, x_{2}^{\prime}\right)}=\left\{\begin{array}{l}\xi_{s_{1} s_{2} s_{1} t_{0}^{-1} s t_{0}^{\prime} s_{2}} \psi\left(\left(\left[x_{1}^{-1}, x_{2}^{\prime-1}\right] x_{2}^{s}\right)^{t_{0}^{\prime} s_{2}}\right) \text { if }\left(t_{0}^{-1} t_{0}^{\prime s}\right)^{s_{1} s_{2} s_{1}} \in T_{3} \\ 0 \text { otherwise. }\end{array}\right.$

$\Xi_{\left(t_{0}, x_{1}, x_{2}, x_{12} ; t_{0}^{\prime}, x_{1}^{\prime}, x_{12}^{\prime}\right)}=\left\{\begin{array}{l}\xi_{s_{1} s_{2} s_{1} t_{0}^{-1} s_{1} s f^{-1} t_{0}^{\prime} s_{1} s_{2}} \psi\left(\left(\left(x_{1}^{\prime-1} x_{1}\right) s_{1} f^{-1}\left(x_{1}^{\prime-1} x_{1}\right)\right)^{t_{0} s_{1} s_{2} s_{1}}\right. \\ \left.\left(\left[x_{2}^{-1}, x_{1} x_{1}^{\prime-1}\right] x_{12}\right)^{s t_{0}^{\prime} s_{1} s_{2}} \omega_{2}^{t_{0} s_{1} s_{2}}\right) \\ \text { if } x_{1}^{\prime} \neq x_{1} \text { and }\left(t_{0}^{-1}\right)^{s_{1} s_{2} s_{1}}\left(f^{-1} t_{0}^{\prime}\right)^{s s_{2} s_{1}} \in T_{3}, \\ \text { where } f \in T \text { such that }\left(x_{1}^{-1} x_{1}^{\prime}\right) f s_{1}\left(x_{1}^{-1} x_{1}^{\prime}\right) \in X_{-1} \backslash\{1\} \\ \text { and } \omega_{2} \in X_{2} \text { such that }\left[\omega_{2}^{-1}, x_{1} x_{1}^{\prime-1}\right]=x_{12}^{\prime} ; \\ 0 \text { otherwise. }\end{array}\right.$

$\Xi_{\left(t_{0}, x_{1}, x_{2} ; t_{0}^{\prime}, x_{2}^{\prime}, x_{12}^{\prime}\right)}=\xi_{s_{1} s_{2} s_{1} t_{0}^{-1} s t_{0}^{\prime} s_{2} s_{1}} \psi\left(\left(x_{12}^{\prime-1}\left[x_{1}^{-1}, x_{2}^{\prime-1}\right]\right)^{s t_{0} s_{1} s_{2}}\left(x_{1} x_{2}\right)^{s t_{0}^{\prime} s_{2} s_{1}}\right)$.

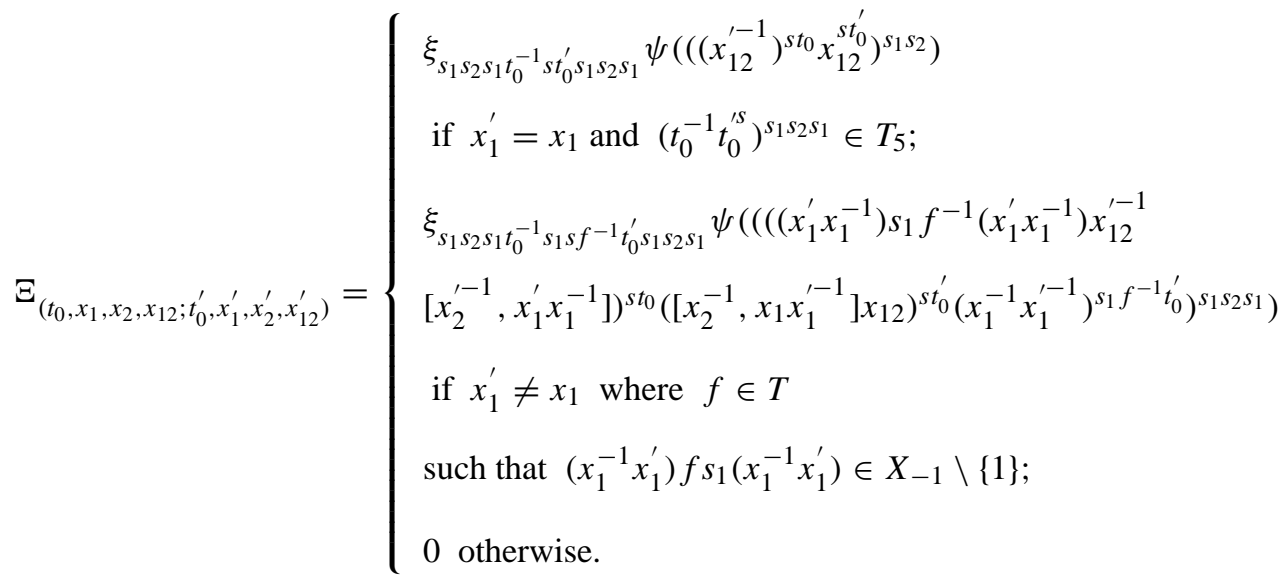

We now determine explicitly the coefficient $\xi_{a}, a \in X_{\psi}$ of the Bessel vector $b$. If $a=u n$, $n \in N_{\psi}, u \in U_{n^{-1}}$, then we know that $\xi_{a}=\xi_{n} \psi\left(u^{-1}\right)$. Thus it is enough to consider only the coefficients $\xi_{n}, n \in N_{\psi}$.

If $\chi$ is the cuspidal character that gives rise to the Bessel vector $b$, then we have

$$
\xi_{n^{-1}}=\chi(\text { ene })=|U|^{-1} \sum_{u \in U} \psi\left(u^{-1}\right) \chi(u t w)
$$


where $n=t w, t \in T, w \in W \cap N_{\psi}$. (We notice that $\xi_{n^{-1}}=\bar{\xi}_{n}$.)

To calculate the value $\chi(u t w)$ we must know which conjugacy class of $\mathrm{GL}_{4}(q)$ contains the element $u t w$.

The conjugacy classes of $\mathrm{GL}_{4}(q)$ are represented by 22 different types of Jordan forms of the elements of $\mathrm{GL}_{4}(K)$, where $K$ is the algebraic closure of the field $\mathbf{F}_{q}$. These types are given in Table 3. Let us note that in this table some of the types of class are empty for small values of $q$. For example, the conjugacy classes of $\mathrm{GL}_{4}(2)$ are classified into the types $C_{1}, C_{2}, C_{3}, C_{4}, C_{5}, C_{15}, C_{16}, C_{18}, C_{19}, C_{21}$ and $C_{22}$, the other types being empty.

Table 3: The conjugacy classes of $G L_{4}(q)$.

\section{Type of Jordan form}

Number of classes

\section{class}

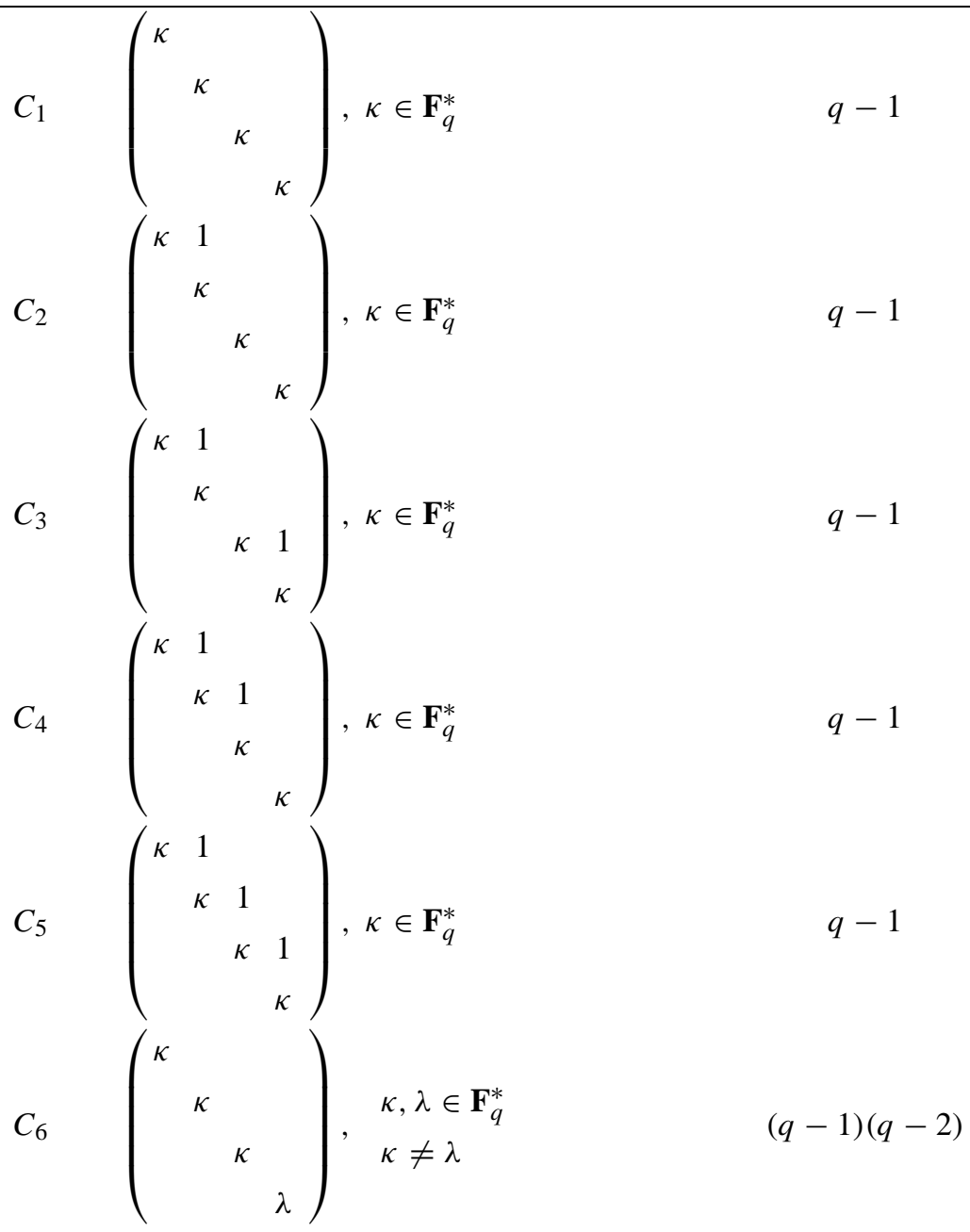

Table 3 continued on next page 


\section{Type of Jordan form}

Number of classes

\section{class}

$C_{7} \quad\left(\begin{array}{cccc}\kappa & 1 & & \\ & \kappa & & \\ & & \kappa & \\ & & \lambda\end{array}\right), \begin{aligned} & \kappa, \lambda \in \mathbf{F}_{q}^{*} \\ & \kappa \neq \lambda\end{aligned}$

$$
(q-1)(q-2)
$$

$C_{8} \quad\left(\begin{array}{cccc}\kappa & 1 & & \\ & \kappa & 1 & \\ & & \kappa & \\ & & & \lambda\end{array}\right), \begin{aligned} & \kappa, \lambda \in \mathbf{F}_{q}^{*} \\ & \kappa \neq \lambda\end{aligned}$

$(q-1)(q-2)$

$C_{9} \quad\left(\begin{array}{llll}\kappa & & & \\ & \kappa & & \\ & & \lambda & \\ & & \lambda\end{array}\right), \begin{aligned} & \kappa, \lambda \in \mathbf{F}_{q}^{*} \\ & \kappa \neq \lambda\end{aligned}$

$\frac{1}{2}(q-1)(q-2)$

$C_{10} \quad\left(\begin{array}{cccc}\kappa & 1 & & \\ & \kappa & & \\ & & \lambda & \\ & & \lambda\end{array}\right), \begin{aligned} & \kappa, \lambda \in \mathbf{F}_{q}^{*} \\ & \kappa \neq \lambda\end{aligned}$

$$
(q-1)(q-2)
$$

$C_{11} \quad\left(\begin{array}{cccc}\kappa & 1 & & \\ & \kappa & & \\ & & \lambda & 1 \\ & & \lambda\end{array}\right), \begin{aligned} & \kappa, \lambda \in \mathbf{F}_{q}^{*} \\ & \kappa \neq \lambda\end{aligned}$

$\frac{1}{2}(q-1)(q-2)$

$C_{12} \quad\left(\begin{array}{cccc}\kappa & & & \\ & \kappa & & \\ & & \lambda & \\ & & \mu\end{array}\right)$,

$\kappa, \lambda, \mu \in \mathbf{F}_{q}^{*}$

$\kappa, \lambda, \mu$ pairwise

$\frac{1}{2}(q-1)(q-2)(q-3)$

different

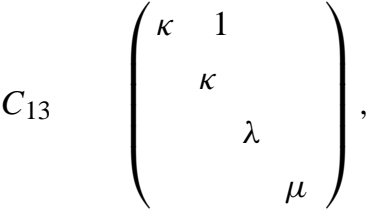

$\kappa, \lambda, \mu \in \mathbf{F}_{q}^{*}$

$\kappa, \lambda, \mu$ pairwise

$\frac{1}{2}(q-1)(q-2)(q-3)$

different

$C_{14} \quad\left(\begin{array}{cccc}\kappa & & & \\ & \lambda & & \\ & & \mu & \\ & & & \\ & & & v\end{array}\right), \begin{array}{lll}\kappa, \lambda, \mu, \nu & \in \mathbf{F}_{q}^{*} \\ & \kappa, \lambda, \mu, v & \text { pairwise } \\ & & \text { different }\end{array}$

$$
\begin{aligned}
\frac{1}{24}(q-1) & (q-2) \\
& (q-3)(q-4)
\end{aligned}
$$

Table 3 continued on next page 
The Cuspidal Modules of the Finite General Linear Groups

Table 3 continued from previous page

\section{Type of Jordan form}

Number of classes

\section{class}

$C_{15} \quad\left(\begin{array}{llll}\kappa & & & \\ & \kappa & & \\ & x & \\ & & x^{q}\end{array}\right), \begin{aligned} & \kappa \in \mathbf{F}_{q}^{*} \\ & x \in \mathbf{F}_{q^{2}} \backslash \mathbf{F}_{q}\end{aligned}$

$\frac{1}{2} q(q-1)^{2}$

$C_{16} \quad\left(\begin{array}{cccc}\kappa & 1 & \\ & \kappa & & \\ & x & \\ & & x^{q}\end{array}\right), \begin{aligned} & \kappa \in \mathbf{F}_{q}^{*} \\ & x \in \mathbf{F}_{q^{2}} \backslash \mathbf{F}_{q}\end{aligned}$

$\frac{1}{2} q(q-1)^{2}$

$C_{17} \quad\left(\begin{array}{llll}\kappa & & & \\ & \lambda & & \\ & & x & \\ & & x^{q}\end{array}\right), \begin{aligned} & \kappa, \lambda \in \mathbf{F}_{q}^{*} \\ & \\ & \end{aligned}$

$\frac{1}{4} q(q-1)^{2}(q-2)$

$C_{18} \quad\left(\begin{array}{cccc}x & & & \\ & x & & \\ & & x^{q} & \\ & & & x^{q}\end{array}\right), x \in \mathbf{F}_{q^{2}} \backslash \mathbf{F}_{q}$

$\frac{1}{2} q(q-1)$

$C_{19} \quad\left(\begin{array}{cccc}x & 1 & & \\ & x & & \\ & & x^{q} & 1 \\ & & & x^{q}\end{array}\right), x \in \mathbf{F}_{q^{2}} \backslash \mathbf{F}_{q}$

$\frac{1}{2} q(q-1)$

$C_{20} \quad\left(\begin{array}{llll}x & & & \\ & & & \\ & x^{q} & & \\ & & y & \\ & & y^{q}\end{array}\right), \begin{array}{lll}x, y \in \mathbf{F}_{q^{2}}^{*} \backslash \mathbf{F}_{q} & \\ x \neq y, y^{q} & & \\ & & \end{array}$

$C_{21} \quad\left(\begin{array}{llll}\kappa & & & \\ & x & & \\ & x^{q} & \\ & & x^{q^{2}}\end{array}\right), \begin{array}{lll}\kappa & \in \mathbf{F}_{q}^{*} \\ & x \in \mathbf{F}_{q^{3}}^{*} \backslash \mathbf{F}_{q} & \frac{1}{3} q(q+1)(q-1)^{2}\end{array}$

$C_{22} \quad\left(\begin{array}{llll}x & & & \\ & x^{q} & & \\ & & x^{q^{2}} & \\ & & & x^{q^{3}}\end{array}\right), x \in \mathbf{F}_{q^{4}} \backslash \mathbf{F}_{q^{2}} \quad \frac{1}{4} q^{2}(q+1)(q-1)$ 
Table 4: The values of the cuspidal character $\chi$.

\begin{tabular}{cl}
\hline Class $C$ & \multicolumn{1}{c}{$\chi(C)$} \\
\hline$C_{1}$ & $\left(q^{3}-1\right)\left(q^{2}-1\right)(q-1) \theta(\kappa)$ \\
$C_{2}$ & $-\left(q^{2}-1\right)(q-1) \theta(\kappa)$ \\
$C_{3}$ & $(q-1) \theta(\kappa)$ \\
$C_{4}$ & $(q-1) \theta(\kappa)$ \\
$C_{5}$ & $-\theta(\kappa)$ \\
$C_{18}$ & $\left(q^{2}-1\right)\left(\theta(x)+\theta\left(x^{q}\right)\right)$ \\
$C_{19}$ & $-\left(\theta(x)+\theta\left(x^{q}\right)\right)$ \\
$C_{22}$ & $-\left(\theta(x)+\theta\left(x^{q}\right)+\theta\left(x^{q^{2}}\right)+\theta\left(x^{q^{3}}\right)\right)$ \\
$C_{i}, i=6,7,8,9,10,11,12$, & 0 \\
$13,14,15,16,17,20,21$ & \\
\hline
\end{tabular}

The values of $\chi$ can be easily obtained using the work of Green [5]. These values are given in Table 4.

Now we have

$$
\chi(\text { etwe })=|U|^{-1} \sum_{C \text { conj.class }} \chi(C) \sum_{\substack{u \in U \\ u t w \in C}} \psi\left(u^{-1}\right) .
$$

To calculate, for a class $C$, the coefficient $\sum_{\substack{u \in U \\ u t w \in C}} \psi\left(u^{-1}\right)$ of $\chi(C)$, we use the same method as in [2]. Thus we have to determine, for a given $n=t w \in N_{\psi}, t \in T, w \in N_{\psi} \cap W$, the elements $u \in U$, such that $u t w \in C$.

For this, first we determine all the pairs $\left(t^{\prime}, u\right)$ so that $u t^{\prime} w \in C$. This can be done by comparing the characteristic polynomial of $u t^{\prime} w$ and that of $C$. Then the use of the minimal polynomial of $C$ and the rank of the matrix $u t^{\prime} w-\lambda I$ gives these pairs, where $\lambda$ is an eigenvalue of the elements in $C$. Having found these pairs, for the given $w \in N_{\psi} \cap W$ we consider only those where $t^{\prime}=t$. These calculations need a considerable amount of work, which cannot be presented here.

Tables 5-11 give for each $w \in N_{\psi} \cap W$ the number of pairs $\left(t^{\prime}, u\right)$ with $u t^{\prime} w \in C$, for every conjugacy class $C$. In these tables we have omitted the classes that do not contain elements of the form $u t^{\prime} w$.

In Tables 9 and 10 we have put $C_{i}^{1}, i=8,11,13,14,16,17,20,21,22$, and $C_{i}^{2}, i=$ $11,13,14,16,17,20$, to denote respectively the conjugacy classes of type: $C_{8}$, with $\lambda=\frac{a^{2}}{\kappa^{3}}$, $a \neq 0, \pm \kappa^{2}, C_{11}$ with $\lambda=-\kappa, C_{13}$ with $\mu=\frac{\kappa^{2}}{\lambda}, \lambda \neq-\kappa, C_{14}$ with $\lambda=-\kappa, v=-\mu$, $\kappa \neq-\mu, C_{16}$ with $\kappa^{2}=x^{q+1}, C_{17}$ with $\kappa \lambda^{\lambda}=x^{q+1}, C_{20}$ with $x^{q+1}=y^{q+1}, C_{21}$ with $-\kappa$ and $-x^{1+q+q^{2}}$ squares in $\mathbf{F}_{q}$ or with $-\kappa$ and $-x^{1+q+q^{2}}$ not squares, $C_{22}$ with 
The Cuspidal Modules of the Finite General Linear Groups

Table 5: $(w=1)$

\begin{tabular}{ccl}
\hline Type of Class & Number of Class & Number of pairs $\left(t^{\prime}, u\right)$ \\
\hline$C_{1}$ & $q-1$ & 1 \\
$C_{2}$ & $q-1$ & $(q-1)\left(3 q^{2}+2 q+1\right)$ \\
$C_{3}$ & $q-1$ & $q(q-1)^{2}(2 q+1)$ \\
$C_{4}$ & $q-1$ & $q^{2}(q-1)^{2}(3 q+1)$ \\
$C_{5}$ & $q-1$ & $q^{3}(q-1)^{3}$ \\
\hline
\end{tabular}

Table 6: $\left(w=w_{1}\right.$ or $\left.w_{2}\right)$

\begin{tabular}{ccl}
\hline Type of Class & Number of Class & Number of pairs $\left(t^{\prime}, u\right)$ \\
\hline$C_{5}$ & $q-1$ & $q^{3}(q-1)$ \\
$C_{8}$ & $(q-1)(q-2)$ & $q^{3}(q-1)$ \\
$C_{11}$ & $\frac{1}{2}(q-1)(q-2)$ & $q^{3}(q-1)$ \\
$C_{13}$ & $\frac{1}{2}(q-1)(q-2)(q-3)$ & $q^{3}(q-1)$ \\
$C_{14}$ & $\frac{1}{24}(q-1)(q-2)(q-3)(q-4)$ & $q^{3}(q-1)$ \\
$C_{16}$ & $\frac{1}{2} q(q-1)^{2}$ & $q^{3}(q-1)$ \\
$C_{17}$ & $\frac{1}{4}(q-1)^{2}(q-2) q$ & $q^{3}(q-1)$ \\
$C_{19}$ & $\frac{1}{2} q(q-1)$ & $q^{3}(q-1)$ \\
$C_{20}$ & $\frac{1}{8}(q+1) q(q-1)(q-2)$ & $q^{3}(q-1)$ \\
$C_{21}$ & $\frac{1}{3}(q+1) q(q-1)^{2}$ & $q^{3}(q-1)$ \\
$C_{22}$ & $\frac{1}{4}(q+1) q^{2}(q-1)$ & $q^{3}(q-1)$ \\
\hline
\end{tabular}

$x^{\frac{1}{2}\left(1+q+q^{2}+q^{3}\right)} \in \mathbf{F}_{q}$ and $C_{11}$ with $\lambda \neq-\kappa, C_{13}$ with $\mu=\frac{a^{2}}{\lambda \kappa^{2}}$, where $a \in \mathbf{F}_{q}^{*} \backslash\left\{ \pm \kappa^{2}, \pm \kappa \lambda\right\}$ such that $\lambda \neq a^{2} \kappa^{-3}, C_{14}$ with $v=\frac{\kappa \lambda}{\mu}, \lambda \neq-\mu, \kappa \neq-\mu, \frac{\mu^{2}}{\lambda}, C_{16}$ with $\kappa^{2} \neq x^{q+1}, C_{17}$ with $\kappa \lambda \neq x^{q+1}, C_{20}$ with $x^{q+1} \neq y^{q+1}$. Moreover $C_{13}^{3}$ and $C_{14}^{3}$ denote respectively the classes of type $C_{13}$ that do not belong to $C_{13}^{1}$, and of $C_{14}$ that do not belong to either $C_{14}^{1}$ or to $C_{14}^{2}$. Finally, $C_{14}^{4}$ denotes the classes of type $C_{14}$ that do not belong to $C_{14}^{2}$.

For each $w \in N_{\psi} \cap W$, we notice, from the above results, that if we multiply, for each type of conjugacy class, the number of classes by the number of pairs, then the sum of these products is equal to

$$
|U| \cdot\left|T w \cap N_{\psi}\right|
$$

as it ought to be.

Now we give the values $\chi\left(\right.$ ene), for $n \in N_{\psi}$. As we have mentioned, the detailed calculations have been worked out by Gotsis [6].

We let $f_{r}(x)=x^{4}+a_{3}(r) x^{3}+a_{2}(r) x^{2}+a_{1}(r) x+a_{0}(r)$ be the characteristic polynomial 
The Cuspidal Modules of the Finite General Linear Groups

Table 7: $\left(w=w_{3}\right.$ or $\left.w_{4}\right)$

\begin{tabular}{ccl}
\hline Type of Class & Number of Class & Number of pairs $\left(t^{\prime}, u\right)$ \\
\hline$C_{4}$ & $q-1$ & $q(q-1)^{2}$ \\
$C_{5}$ & $q-1$ & $q^{3}(q-1)^{2}$ \\
$C_{7}$ & $(q-1)(q-2)$ & $q(q-1)^{2}$ \\
$C_{8}$ & $(q-1)(q-2)$ & $q^{2}(q-1)^{2}(q+1)$ \\
$C_{10}$ & $(q-1)(q-2)$ & $q(q-1)^{2}$ \\
$C_{11}$ & $\frac{1}{2}(q-1)(q-2)$ & $q(q-1)^{2}\left(q^{2}+q-1\right)$ \\
$C_{12}$ & $\frac{1}{2}(q-1)(q-2)(q-3)$ & $q(q-1)^{2}$ \\
$C_{13}$ & $\frac{1}{2}(q-1)(q-2)(q-3)$ & $q^{2}(q-1)^{2}(q+2)$ \\
$C_{14}$ & $\frac{1}{24}(q-1)(q-2)(q-3)(q-4)$ & $q(q-1)^{2}\left(q^{2}+3 q+1\right)$ \\
$C_{15}$ & $\frac{1}{2} q(q-1)^{2}$ & $q(q-1)^{2}$ \\
$C_{16}$ & $\frac{1}{2} q(q-1)^{2}$ & $q^{3}(q-1)^{2}$ \\
$C_{17}$ & $\frac{1}{4} q(q-1)^{2}(q-2)$ & $q(q-1)^{2}\left(q^{2}+q+1\right)$ \\
$C_{19}$ & $\frac{1}{2} q(q-1)$ & $q(q-1)^{2}\left(q^{2}-q+1\right)$ \\
$C_{20}$ & $\frac{1}{8}(q+1) q(q-1)(q-2)$ & $q(q-1)^{2}\left(q^{2}-q+1\right)$ \\
$C_{21}$ & $\frac{1}{3}(q+1) q(q-1)^{2}$ & $q(q-1)^{2}\left(q^{2}+1\right)$ \\
$C_{22}$ & $\frac{1}{4}(q+1) q^{2}(q-1)$ & $q(q-1)^{2}\left(q^{2}-q+1\right)$ \\
\hline
\end{tabular}

of an element $r$ of the torus $T_{4}$, which is identified with $\mathbf{F}_{q^{4}}^{*}$. In what follows we put

$$
u=\left(\begin{array}{llll}
1 & a & \delta & \zeta \\
0 & 1 & \beta & \varepsilon \\
0 & 0 & 1 & \gamma \\
0 & 0 & 0 & 1
\end{array}\right)
$$

For $w=w_{0}$ and $t=\left(\begin{array}{cccc}\mu & & & \\ & v & & \\ & & \rho & \\ & & & \tau\end{array}\right)$, we consider the function

$$
F_{0}(r, t)=\left\{\begin{array}{l}
-F_{0}^{\prime}(r, t)+q^{-5} \sum_{a \in \mathbf{F}_{q}^{*}} \psi\left(-a+\frac{\mu\left(r^{2}+v \rho\right)^{2}}{a v r^{4}}-\frac{r^{2}-v \rho}{\rho r}\right) \\
\quad \text { if } r \in \mathbf{F}_{q}^{*}, \\
-F_{0}^{\prime}(r, t)+q^{-4} \psi\left(v \frac{1+r^{q-1}}{r^{q}}\right) \text { if } r \in \mathbf{F}_{q^{2}} \backslash \mathbf{F}_{q} \text { and } v \rho=-r^{q+1}, \\
-F_{0}^{\prime}(r, t) \text { otherwise, }
\end{array}\right.
$$

where 
The Cuspidal Modules of the Finite General Linear Groups

Table 8: $\left(w=w_{5}\right)$

\begin{tabular}{ccl}
\hline Type of Class & Number of Class & Number of pairs $\left(t^{\prime}, u\right)$ \\
\hline$C_{2}$ & $q-1$ & $q-1$ \\
$C_{3}$ & $q-1$ & $(q-1)^{2}$ \\
$C_{4}$ & $q-1$ & $4 q(q-1)^{2}$ \\
$C_{5}$ & $q-1$ & $q(q-1)^{3}(q+2)$ \\
$C_{6}$ & $(q-1)(q-2)$ & $q-1$ \\
$C_{7}$ & $(q-1)(q-2)$ & $(q-1)^{2}(4 q+1)$ \\
$C_{8}$ & $(q-1)(q-2)$ & $q(q-1)^{2}\left(q^{2}+3 q-2\right)$ \\
$C_{10}$ & $(q-1)(q-2)$ & $q(q-1)(3 q-2)$ \\
$C_{11}$ & $\frac{1}{2}(q-1)(q-2)$ & $q(q-1)^{2}\left(q^{2}+4 q-3\right)$ \\
$C_{12}$ & $\frac{1}{2}(q-1)(q-2)(q-3)$ & $q(q-1)(3 q-2)$ \\
$C_{13}$ & $\frac{1}{2}(q-1)(q-2)(q-3)$ & $q(q-1)^{2}\left(q^{2}+5 q-1\right)$ \\
$C_{14}$ & $\frac{1}{24}(q-1)(q-2)(q-3)(q-4)$ & $q(q-1)^{2}\left(q^{2}+6 q+1\right)$ \\
$C_{15}$ & $\frac{1}{2} q(q-1)^{2}$ & $q(q-1)(3 q-2)$ \\
$C_{16}$ & $\frac{1}{2} q(q-1)^{2}$ & $q(q-1)^{2}\left(q^{2}+q-1\right)$ \\
$C_{17}$ & $\frac{1}{4} q(q-1)^{2}(q-2)$ & $q(q-1)^{2}(q+1)^{2}$ \\
$C_{19}$ & $\frac{1}{2} q(q-1)$ & $q(q-1)^{4}$ \\
$C_{20}$ & $\frac{1}{8}(q+1) q(q-1)(q-2)$ & $q(q-1)^{4}$ \\
$C_{21}$ & $\frac{1}{3}(q+1) q(q-1)^{2}$ & $q(q-1)^{2}\left(q^{2}+1\right)$ \\
$C_{22}$ & $\frac{1}{4}(q+1) q^{2}(q-1)$ & $q(q-1)^{4}$ \\
\hline & &
\end{tabular}

$$
\begin{aligned}
F_{0}^{\prime}(r, t)= & q^{-6} \sum_{\substack{a, \beta, \delta \in \mathbf{F}_{q} \\
a^{2} v+a \beta \delta \rho-\delta^{2} \rho \neq 0}} \psi\left(-a-\beta+\mu\left(a^{2} v+a \beta \delta \rho-\delta^{2} \rho\right)^{-1} a_{0}(r)^{-1} v^{-1}\left(a_{0}(r)\right.\right. \\
& (a v+\beta \delta \rho)-a_{1}(r) \delta \nu \rho+a_{2}(r) a v^{2} \rho+a_{3}(r) v^{2} \rho^{2}(a \beta-\delta) \\
& \left.\left.+v^{2} \rho^{2}\left(a \beta^{2} \rho+a v-\beta \delta \rho\right)\right)\right) .
\end{aligned}
$$

Then $\chi\left(e t w_{0} e\right)=\sum_{\substack{r \in T_{4} \\ \operatorname{det} r=\operatorname{det} t}} F_{0}(r, t) \theta(r)$, where det $r=a_{0}(r)=r^{1+q+q^{2}+q^{3}}$.

For $w=w_{1}$ and $t=\left(\begin{array}{cccc}\mu & & & \\ & v & & \\ & & & \\ & & & \\ & & & v\end{array}\right)$ we put $F_{1}(r, t)=-q^{-3} \psi\left(\frac{a_{3}(r)}{v}\right)$. Then

$$
\chi\left(e t w_{1} e\right)=\sum_{\substack{r \in T_{4} \\ \operatorname{det} r=-\operatorname{det} t}} F_{1}(r, t) \theta(r)
$$


The Cuspidal Modules of the Finite General Linear Groups

Table 9: $\left(w=w_{6}, \quad \operatorname{char} \mathbf{F}_{q} \neq 2\right)$

\begin{tabular}{|c|c|c|}
\hline Type of Class & Number of Class & Number of pairs $\left(t^{\prime}, u\right)$ \\
\hline$C_{3}$ & $q-1$ & $q(q-1)$ \\
\hline$C_{4}$ & $q-1$ & $q(q-1)^{2}$ \\
\hline$C_{5}$ & $q-1$ & $3 q^{2}(q-1)^{2}$ \\
\hline$C_{8}^{1}$ & $\frac{1}{2}(q-1)(q-3)$ & $2 q^{2}(q-1)^{2}$ \\
\hline$C_{9}$ & $\frac{1}{2}(q-1)(q-2)$ & $q(q-1)$ \\
\hline$C_{10}$ & $(q-1)(q-2)$ & $q(q-1)^{2}$ \\
\hline$C_{11}^{1}$ & $\frac{1}{2}(q-1)$ & $q(q-1)\left(5 q^{2}-4 q+1\right)$ \\
\hline$C_{11}^{2}$ & $\frac{1}{2}(q-1)(q-3)$ & $q(q-1)^{2}(3 q-1)$ \\
\hline$C_{13}^{1}$ & $\frac{1}{2}(q-1)(q-3)$ & $2 q^{2}(q-1)(2 q-1)$ \\
\hline$C_{13}^{2}$ & $\frac{1}{4}(q-1)(q-3)(q-5)$ & $2 q^{2}(q-1)^{2}$ \\
\hline$C_{14}^{1}$ & $\frac{1}{8}(q-1)(q-3)$ & $2 q^{2}(q-1)(3 q-1)$ \\
\hline$C_{14}^{2}$ & $\frac{1}{8}(q-1)(q-3)(q-5)$ & $2 q^{2}(q-1)(2 q-1)$ \\
\hline$C_{14}^{3}$ & $\frac{1}{48}(q-1)(q-3)(q-5)(q-7)$ & $2 q^{2}(q-1)^{2}$ \\
\hline$C_{16}^{1}$ & $\frac{1}{2}(q-1)^{2}$ & $2 q^{2}(q-1)(2 q-1)$ \\
\hline$C_{16}^{2}$ & $\frac{1}{4}(q-1)^{2}(q-3)$ & $2 q^{2}(q-1)^{2}$ \\
\hline$C_{17}^{1}$ & $\frac{1}{4}(q-1)^{3}$ & $2 q^{2}(q-1)(2 q-1)$ \\
\hline$C_{17}^{2}$ & $\frac{1}{8}(q-1)^{3}(q-3)$ & $2 q^{2}(q-1)^{2}$ \\
\hline$C_{18}$ & $\frac{1}{2} q(q-1)$ & $q(q-1)$ \\
\hline$C_{19}$ & $\frac{1}{2} q(q-1)$ & $q(q-1)^{2}(3 q+1)$ \\
\hline$C_{20}^{1}$ & $\frac{1}{8}(q-1)^{3}$ & $2 q^{2}(q-1)(2 q-1)$ \\
\hline$C_{20}^{2}$ & $\frac{1}{16}(q-1)(q-3)\left(q^{2}+1\right)$ & $2 q^{2}(q-1)^{2}$ \\
\hline$C_{21}^{1}$ & $\frac{1}{6}(q+1) q(q-1)^{2}$ & $2 q^{2}(q-1)^{2}$ \\
\hline$C_{22}^{1}$ & $\frac{1}{8}(q+1)^{2}(q-1)^{2}$ & $2 q^{2}(q-1)^{2}$ \\
\hline
\end{tabular}


The Cuspidal Modules of the Finite General Linear Groups

Table 10: $\left(w=w_{6}, \quad \operatorname{char} \mathbf{F}_{q}=2\right)$

\begin{tabular}{ccl}
\hline Type of Class & Number of Class & Number of pairs $\left(t^{\prime}, u\right)$ \\
\hline$C_{3}$ & $q-1$ & $q(q-1)$ \\
$C_{4}$ & $q-1$ & $q(q-1)^{2}$ \\
$C_{5}$ & $q-1$ & $2 q^{2}(q-1)^{2}$ \\
$C_{8}$ & $(q-1)(q-2)$ & $q^{2}(q-1)^{2}$ \\
$C_{9}$ & $\frac{1}{2}(q-1)(q-2)$ & $q^{2}(q-1)$ \\
$C_{11}$ & $\frac{1}{2}(q-1)(q-2)$ & $2 q^{2}(q-1)^{2}$ \\
$C_{13}^{1}$ & $\frac{1}{2}(q-1)(q-2)$ & $q^{2}(q-1)(3 q-1)$ \\
$C_{13}^{3}$ & $\frac{1}{2}(q-1)(q-2)(q-4)$ & $q^{2}(q-1)^{2}$ \\
$C_{14}^{2}$ & $\frac{1}{8}(q-1)(q-3)(q-4)$ & $q^{2}(q-1)(3 q-1)$ \\
$C_{14}^{4}$ & $\frac{1}{24}(q-1)(q-2)(q-4)(q-6)$ & $q^{2}(q-1)^{2}$ \\
$C_{16}^{1}$ & $\frac{1}{2} q(q-1)$ & $q^{2}(q-1)(3 q-1)$ \\
$C_{16}^{2}$ & $\frac{1}{2} q(q-1)(q-2)$ & $q^{2}(q-1)^{2}$ \\
$C_{17}^{1}$ & $\frac{1}{4} q(q-1)(q-2)$ & $q^{2}(q-1)(3 q-1)$ \\
$C_{17}^{2}$ & $\frac{1}{4} q(q-1)(q-2)^{2}$ & $q^{2}(q-1)^{2}$ \\
$C_{18}$ & $\frac{1}{2} q(q-1)$ & $q(q-1)$ \\
$C_{19}$ & $\frac{1}{2} q(q-1)$ & $q(q-1)^{2}(2 q+1)$ \\
$C_{20}^{1}$ & $\frac{1}{8} q(q-1)(q-2)$ & $q^{2}(q-1)(3 q-1)$ \\
$C_{20}^{2}$ & $\frac{1}{8} q^{2}(q-1)(q-2)$ & $q^{2}(q-1)^{2}$ \\
$C_{21}$ & $\frac{1}{3}(q+1) q(q-1)^{2}$ & $q^{2}(q-1)^{2}$ \\
$C_{22}$ & $\frac{1}{4}(q+1) q^{2}(q-1)$ & $q^{2}(q-1)^{2}$ \\
\hline & &
\end{tabular}

For $w=w_{2}$ and $t=\left(\begin{array}{cccc}\mu & & & \\ & \mu & & \\ & & \mu & \\ & & & v\end{array}\right)$ we have $\chi\left(e t w_{2} e\right)=\overline{\chi\left(e t^{\prime} w_{1} e\right)}$, where

$$
t^{\prime}=\left(\begin{array}{cccc}
v^{-1} & & & \\
& \mu^{-1} & & \\
& & \mu^{-1} & \\
& & & \mu^{-1}
\end{array}\right)
$$


The Cuspidal Modules of the Finite General Linear Groups

Table 11: $\left(w=w_{0}\right)$

\begin{tabular}{|c|c|c|}
\hline $\begin{array}{c}\text { Type } \\
\text { of Class }\end{array}$ & $\begin{array}{l}\text { Number } \\
\text { of Class }\end{array}$ & $\begin{array}{c}\text { Number } \\
\text { of Pairs }\left(t^{\prime}, u\right)\end{array}$ \\
\hline$C_{3}$ & $q-1$ & $(q-1)^{2}$ \\
\hline$C_{4}$ & $q-1$ & $q(q-1)^{3}$ \\
\hline$C_{5}$ & $q-1$ & $q^{3}(q-1)^{3}$ \\
\hline$C_{7}$ & $(q-1)(q-2)$ & $(q-1)^{2}\left(q^{2}+1\right)$ \\
\hline$C_{8}$ & $(q-1)(q-2)$ & $q^{2}(q-1)^{3}(q+1)$ \\
\hline$C_{9}$ & $\frac{1}{2}(q-1)(q-2)$ & $(q-1)^{2}$ \\
\hline$C_{10}$ & $(q-1)(q-2)$ & $q(q-1)^{3}$ \\
\hline$C_{11}$ & $\frac{1}{2}(q-1)(q-2)$ & $q(q-1)^{2}\left(q^{3}-q+2\right)$ \\
\hline$C_{12}$ & $\frac{1}{2}(q-1)(q-2)(q-3)$ & $(q-1)^{2}\left(q^{2}+1\right)$ \\
\hline$C_{13}$ & $\frac{1}{2}(q-1)(q-2)(q-3)$ & $q(q-1)^{2}\left(q^{3}+q^{2}-q+1\right)$ \\
\hline$C_{14}$ & $\frac{1}{24}(q-1)(q-2)(q-3)(q-4)$ & $(q-1)^{2}\left(q^{4}+2 q^{3}+2 q+1\right)$ \\
\hline$C_{15}$ & $\frac{1}{2} q(q-1)^{2}$ & $(q-1)^{4}$ \\
\hline$C_{16}$ & $\frac{1}{2} q(q-1)^{2}$ & $q(q-1)^{2}\left(q^{3}-q^{2}+q+1\right)$ \\
\hline$C_{17}$ & $\frac{1}{4} q(q-1)^{2}(q-2)$ & $(q-1)^{2}\left(q^{4}+1\right)$ \\
\hline$C_{18}$ & $\frac{1}{2} q(q-1)$ & $(q-1)^{2}$ \\
\hline$C_{19}$ & $\frac{1}{2} q(q-1)$ & $q(q-1)^{3}\left(q^{2}-q+2\right)$ \\
\hline$C_{20}$ & $\frac{1}{8}(q+1) q(q-1)(q-2)$ & $(q-1)^{2}\left(q^{4}-2 q^{3}+4 q^{2}-2 q+1\right)$ \\
\hline$C_{21}$ & $\frac{1}{3}(q+1) q(q-1)^{2}$ & $(q-1)^{4}\left(q^{2}+q+1\right)$ \\
\hline$C_{22}$ & $\frac{1}{4}(q+1) q^{2}(q-1)$ & $(q-1)^{4}\left(q^{2}+1\right)$ \\
\hline
\end{tabular}

For $w=w_{3}$ and $t=\left(\begin{array}{cccc}\mu & & & \\ & v & & \\ & & \rho & \\ & & & \rho\end{array}\right)$ we have $\chi\left(\right.$ et $\left.w_{3} e\right)=\overline{\chi\left(e t^{\prime} w_{4} e\right)}$, where

$$
t^{\prime}=\left(\begin{array}{cccc}
\rho^{-1} & & & \\
& \rho^{-1} & & \\
& & v^{-1} & \\
& & & \mu^{-1}
\end{array}\right)
$$


The Cuspidal Modules of the Finite General Linear Groups

For $w=w_{4}$ and $t=\left(\begin{array}{cc}\mu & \\ \mu & \\ v & \\ & \rho\end{array}\right)$ we consider the function
\[ F_{4}(r, t)=\left\{\begin{array}{l}-F_{4}^{\prime}(r, t)+q^{-4} \psi\left(\frac{-r^{3}+2 \mu v^{2}}{r^{2} v}\right) \text { if } r \in \mathbf{F}_{q}^{*}, \\ -F_{4}^{\prime}(r, t) \text { otherwise, }\end{array}\right. \]

where

$$
\begin{aligned}
F_{4}^{\prime}(r, t)= & q^{-5} \sum_{\substack{\beta, \delta \in \mathbf{F}_{q} \\
\beta \delta v+\mu \neq 0}} \psi\left(a _ { 0 } ( r ) ^ { - 1 } ( \beta \delta v + \mu ) ^ { - 1 } \left(-a_{3}(r) \beta \mu^{2} v^{2}-a_{2}(r) \mu^{2} v+a_{1}(r) \delta \mu \nu\right.\right. \\
& \left.\left.-a_{0}(r)\left(\beta^{2} \delta v+\beta \mu+\delta^{2} v\right)-\beta^{2} \mu^{2} v^{3}\right)\right) .
\end{aligned}
$$

Then $\chi\left(\right.$ etw $\left.w_{4} e\right)=\sum_{\substack{r \in T_{4} \\ \operatorname{det} r=-\operatorname{det} t}} F_{4}(r, t) \theta(r)$.

For $w=w_{5}$ and $t=\left(\begin{array}{cccc}\mu & & & \\ & v & & \\ & & v & \\ & & & \rho\end{array}\right)$ we consider the function

$$
F_{5}(r, t)=\left\{\begin{array}{l}
-\left(q^{-5}+q^{-4}+q^{-3}\right) \text { if } r=v \\
-q^{-5} \sum_{a, \beta \in \mathbf{F}_{q}^{*}} \psi\left(-a-\beta-\frac{f_{r}(\nu) \mu}{a_{0}(r) a \beta}\right) \text { otherwise. }
\end{array}\right.
$$

Then $\chi\left(\right.$ etw $\left.w_{5} e\right)=\sum_{\substack{r \in T_{4} \\ \operatorname{det} r=-\operatorname{det} t}} F_{5}(r, t) \theta(r)$.

For $w=w_{6}$ and $t=\left(\begin{array}{cccc}\mu & & & \\ & \mu & & \\ & & & \\ & & & v\end{array}\right)$, we put $F_{6}(r, t)$ for the function

$$
F_{6}(r, t)=-q^{-4}\left(F_{6}^{\prime}(r, t)+\sum_{\beta \in \mathbf{F}_{q}^{*}} \psi\left(-\beta+\frac{a_{1}(r)+a_{3}(r) \mu \nu}{\beta \mu \nu^{2}}\right)\right),
$$

where $F_{6}^{\prime}(r, t)=\left\{\begin{array}{l}-q \text { if } r \in \mathbf{F}_{q^{2}} \backslash \mathbf{F}_{q} \text { and } \mu \nu=-r^{q+1}, \\ 0 \text { otherwise. }\end{array}\right.$

Then $\chi\left(e t w_{6} e\right)=\sum_{r \in T_{4}} F_{6}(r, t) \theta(r)$.

Finally, let $w=1$ and $t \in Z$. Easily, we have

$$
\chi(\text { ete })=\theta(t) .
$$


This is a consequence of Burnside's Theorem, which states that the linear span of the images of the elements of a group $G$, under an irreducible representation $R$, is the full matrix algebra of all $k \times k$ complex matrices, where $k$ is the degree of $R$. Combining Burnside's Theorem with the fact that a cuspidal character of $\mathrm{GL}_{n}(q)$ has degree $\left(q^{n-1}-1\right) \cdots(q-1)$, we get $\chi(e t e)=\theta(t)$, for $t \in Z$.

We conclude the paper by giving the action of the affine subgroup $A$ of $\mathrm{GL}_{4}(q)$ on $\mathbb{C} G b$. As we have seen this is a monomial action. Subgroup $A$ is generated by the subgroups $T_{0}, X_{1}, X_{2}$ and $X_{3}$, and the Weyl elements $s_{1}$ and $s_{2}$. Below, we give the action of $T_{0}, X_{1}, X_{2}, X_{3}, s_{1}$ and $s_{2}$ on the basis $\{y b \mid y \in Y\}$ of $\mathbb{C} G b$.

The action of $t_{0}^{\prime} \in T_{0}$

$t_{0}^{\prime}\left(t_{0} b\right)=\left(t_{0}^{\prime} t_{0}\right) b$

$t_{0}^{\prime}\left(x_{1} t_{0} s_{1} b\right)=x_{1,-1}^{t_{0}^{-1}}\left(t_{0}^{\prime} t_{0}\right) s_{1} b$

$t_{0}^{\prime}\left(x_{2} t_{0} s_{2} b\right)=x_{2}^{t_{0}^{\prime-1}}\left(t_{0}^{\prime} t_{0}\right) s_{2} b$

$t_{0}^{\prime}\left(x_{1} x_{12} t_{0} s_{1} s_{2} b\right)=x_{1,-1}^{t_{0}^{\prime-1}} x_{12-1}^{t^{\prime-1}}\left(t_{0}^{\prime} t_{0}\right) s_{1} s_{2} b$

$t_{0}^{\prime}\left(x_{2} x_{12} t_{0} s_{2} s_{1} b\right)=x_{2}^{t_{0}^{\prime-1}} x_{12,1}^{t_{0}^{\prime}}\left(\underset{1}{t_{0}^{\prime}} t_{0}\right) s_{2} s_{1} b$

$t_{0}^{\prime}\left(x_{1} x_{2} x_{12} t_{0} s_{1} s_{2} s_{1} b\right)=x_{1}^{t_{0}^{\prime-1}} \quad x_{2}^{t_{0}^{\prime-1}} \quad x_{12}^{t_{0}^{\prime-1}}\left(t_{0}^{\prime} t_{0}\right) s_{1} s_{2} s_{1} b$

\section{The action of $x_{1}^{\prime} \in X_{1}$}

We notice first that we have the following relations:

(i) $x_{1}^{\prime}\left(x_{2} t_{0} s_{2}\right)=x_{2} t_{0} s_{2} x_{1}^{\prime t_{0} s_{2}}\left[x_{1}^{\prime-1}, x_{2}^{-1}\right]^{t_{0} s_{2}}$, with $x_{1}^{\prime t_{0} s_{2}} \in X_{12}$ and $\left[x_{1}^{\prime-1}, x_{2}^{-1}\right]^{t_{0} s_{2}} \in X_{1}$.

(ii) $x_{1}^{\prime}\left(x_{2} x_{12} t_{0} s_{2} s_{1}\right)=x_{2}\left(\left[x_{1}^{\prime-1}, x_{2}^{-1}\right] x_{12}\right) t_{0} s_{2} s_{1} x_{1}^{\prime t_{0} s_{2} s_{1}}$, with $\left[x_{1}^{\prime-1}, x_{2}^{-1}\right] x_{12} \in X_{12}$ and $x_{1}^{\prime t_{0} s_{2} s_{1}} \in X_{2}$.

The action of $x_{1}^{\prime} \in X_{1}$ is given as follows:

$x_{1}^{\prime}\left(t_{0} b\right)=\psi\left(x_{1}^{\prime t_{0}}\right)\left(t_{0} b\right)$

$x_{1}^{\prime}\left(x_{1} t_{0} s_{1} b\right)=\left(x_{1}^{\prime} x_{1}\right) t_{0} s_{1} b$

$x_{1}^{\prime}\left(x_{2} t_{0} s_{2} b\right)=\psi\left(\left[x_{1}^{\prime-1}, x_{2}^{-1}\right]^{t_{0} s_{2}}\right)\left(x_{2} t_{0} s_{2} b\right)$ by (i)

$x_{1}^{\prime}\left(x_{1} x_{12} t_{0} s_{1} s_{2} b\right)=\left(x_{1}^{\prime} x_{1}\right) x_{12} t_{0} s_{1} s_{2} b$

$x_{1}^{\prime}\left(x_{2} x_{12} t_{0} s_{2} s_{1} b\right)=\psi\left(x_{1}^{\prime t_{0} s_{2} s_{1}}\right)\left(x_{2}\left(\left[x_{1}^{\prime-1}, x_{2}^{-1}\right] x_{12}\right) t_{0} s_{2} s_{1} b\right)$ by (ii)

$x_{1}^{\prime}\left(x_{1} x_{2} x_{12} t_{0} s_{1} s_{2} s_{1} b\right)=\left(x_{1}^{\prime} x_{1}\right) x_{2} x_{12} t_{0} s_{1} s_{2} s_{1} b$.

\section{The action of $x_{2}^{\prime} \in X_{2}$}

We have the following relations:

(i) $x_{2}^{\prime}\left(x_{1} t_{0} s_{1}\right)=x_{1} t_{0} s_{1} x_{2}^{\prime t_{0} s_{1}}\left[x_{2}^{\prime-1}, x_{1}^{-1}\right]^{t_{0} s_{1}}$, with $x_{2}^{\prime t_{0} s_{1}} \in X_{12}$ and $\left[x_{2}^{\prime-1}, x_{1}^{-1}\right]^{t_{0} s_{1}} \in X_{2}$.

(ii) $x_{2}^{\prime}\left(x_{1} x_{12} t_{0} s_{1} s_{2}\right)=x_{1}\left(\left[x_{2}^{\prime-1}, x_{1}^{-1}\right] x_{12}\right) t_{0} s_{1} s_{2} x_{2}^{\prime t_{0} s_{1} s_{2}}$, with $\left[x_{2}^{\prime-1}, x_{1}^{-1}\right] x_{12} \in X_{12}$ and $x_{2}^{\prime t_{0} s_{1} s_{2}} \in X_{1}$.

(iii) $x_{2}^{\prime}\left(x_{1} x_{2} x_{12} t_{0} s_{1} s_{2} s_{1}\right)=x_{1}\left(x_{2}^{\prime} x_{2}\right)\left(\left[x_{2}^{\prime-1}, x_{1}^{-1}\right] x_{12}\right) t_{0} s_{1} s_{2} s_{1}$, with $\left[x_{2}^{\prime-1}, x_{1}^{-1}\right] x_{12} \in X_{12}$.

The action of $x_{2}^{\prime} \in X_{2}$ is as follows: 
$x_{2}^{\prime}\left(t_{0} b\right)=\psi\left(x_{2}^{\prime t_{0}}\right)\left(t_{0} b\right)$

$x_{2}^{\prime}\left(x_{1} t_{0} s_{1} b\right)=\psi\left(\left[x_{2}^{\prime-1}, x_{1}^{-1}\right]^{t_{0} s_{1}}\right)\left(x_{1} t_{0} s_{1} b\right)$ by (i)

$x_{2}^{\prime}\left(x_{2} t_{0} s_{2} b\right)=\left(x_{2}^{\prime} x_{2}\right) t_{0} s_{2} b$

$x_{2}^{\prime}\left(x_{1} x_{12} t_{0} s_{1} s_{2} b\right)=\psi\left(x_{2}^{\prime t_{0} s_{1} s_{2}}\right)\left(x_{1}\left(\left[x_{2}^{\prime-1}, x_{1}^{-1}\right] x_{12}\right) t_{0} s_{1} s_{2} b\right)$ by (ii)

$x_{2}^{\prime}\left(x_{2} x_{12} t_{0} s_{2} s_{1} b\right)=\left(x_{2}^{\prime} x_{2}\right) x_{12} t_{0} s_{2} s_{1} b$

$x_{2}^{\prime}\left(x_{1} x_{2} x_{12} t_{0} s_{1} s_{2} s_{1} b\right)=x_{1}\left(x_{2}^{\prime} x_{2}\right)\left(\left[x_{2}^{\prime-1}, x_{1}^{-1}\right] x_{12}\right) t_{0} s_{1} s_{2} s_{1} b$ by (iii).

The action of $x_{3}^{\prime} \in X_{3}$

The relations we need here are the following:

(i) $x_{3}^{\prime}\left(x_{2} t_{0} s_{2}\right)=x_{2} t_{0} s_{2} x_{3}^{\prime t_{0} s_{2}}\left[x_{3}^{\prime-1}, x_{2}^{-1}\right]^{t_{0} s_{2}}$, with $x_{3}^{\prime t_{0} s_{2}} \in X_{23}$ and $\left[x_{3}^{\prime-1}, x_{2}^{-1}\right]^{t_{0} s_{2}} \in X_{3}$.

(ii) $x_{3}^{\prime}\left(x_{1} x_{12} t_{0} s_{1} s_{2}\right)=x_{1} x_{12} t_{0} s_{1} s_{2} x_{3}^{\prime t_{0} s_{2}}\left[x_{3}^{\prime-1}, x_{12}^{-1}\right]^{t_{0} s_{1} s_{2}}$, with $x_{3}^{\prime t_{0} s_{2}} \in X_{23}$ and $\left[x_{3}^{\prime-1}, x_{12}^{-1}\right]^{t_{0} s_{1} s_{2}} \in X_{3}$.

(iii) $x_{3}^{\prime}\left(x_{2} x_{12} t_{0} s_{2} s_{1}\right)=x_{2} x_{12} t_{0} s_{2} s_{1} x_{3}^{\prime t_{0} s_{2} s_{1}}\left[x_{3}^{\prime-1}, x_{12}^{-1}\right]^{t_{0} s_{2} s_{1}}\left[x_{3}^{\prime-1}, x_{2}^{-1}\right]^{t_{0} s_{2}}$, with $x_{3}^{1 t_{0} s_{2} s_{1}} \in X_{123},\left[x_{3}^{\prime-1}, x_{12}^{-1}\right]^{t_{0} s_{2} s_{1}} \in X_{23}$ and $\left[x_{3}^{\prime-1}, x_{2}^{-1}\right]^{t_{0} s_{2}} \in X_{3}$.

(iv) $x_{3}^{\prime}\left(x_{1} x_{2} x_{12} t_{0} s_{1} s_{2} s_{1}\right)=x_{1} x_{2} x_{12} t_{0} s_{1} s_{2} s_{1} x_{3}^{\prime t_{0} s_{2} s_{1}}\left[x_{3}^{\prime-1}, x_{12}^{-1}\right]^{t_{0} s_{1} s_{2}}\left[x_{3}^{\prime-1}, x_{2}^{-1}\right]^{t_{0}}$, with $x_{3}^{1 t_{0} s_{2} s_{1}} \in X_{123},\left[x_{3}^{\prime-1}, x_{12}^{-1}\right]^{t_{0} s_{1} s_{2}} \in X_{3}$ and $\left[x_{3}^{\prime-1}, x_{2}^{-1}\right]^{t_{0}} \in X_{23}$.

The action of $x_{3}^{\prime} \in X_{3}$ is given by

$x_{3}^{\prime}\left(t_{0} b\right)=\psi\left(x_{3}^{\prime t_{0}}\right)\left(t_{0} b\right)$

$x_{3}^{\prime}\left(x_{1} t_{0} s_{1} b\right)=\psi\left(x_{3}^{\prime t_{0}}\right)\left(x_{1} t_{0} s_{1} b\right)$

$x_{3}^{\prime}\left(x_{2} t_{0} s_{2} b\right)=\psi\left(\left[x_{3}^{\prime-1}, x_{2}^{-1}\right]^{t_{0} s_{2}}\right)\left(x_{2} t_{0} s_{2} b\right)$ by (i)

$x_{3}^{\prime}\left(x_{1} x_{12} t_{0} s_{1} s_{2} b\right)=\psi\left(\left[x_{3}^{\prime-1}, x_{12}^{-1}\right]^{t_{0} s_{1} s_{2}}\right)\left(x_{1} x_{12} t_{0} s_{1} s_{2} b\right)$ by (ii)

$x_{3}^{\prime}\left(x_{2} x_{12} t_{0} s_{2} s_{1} b\right)=\psi\left(\left[x_{3}^{\prime-1}, x_{2}^{-1}\right]^{t_{0} s_{2}}\right)\left(x_{2} x_{12} t_{0} s_{2} s_{1} b\right)$ by (iii)

$x_{3}^{\prime}\left(x_{1} x_{2} x_{12} t_{0} s_{1} s_{2} s_{1} b\right)=\psi\left(\left[x_{3}^{\prime-1}, x_{12}^{-1}\right]^{t_{0} s_{1} s_{2}}\right)\left(x_{1} x_{2} x_{12} t_{0} s_{1} s_{2} s_{1} b\right)$ by (iv).

\section{The action of $s_{1}$}

We have the following relations:

(i) $s_{1}\left(x_{1} t_{0} s_{1}\right)=\left\{\begin{array}{l}t_{0}^{s_{1}} \text { if } x_{1}=1 ; \\ y_{1}\left(t_{0}^{\prime} t_{0}\right) s_{1} y_{1}^{s_{1} t_{0} s_{1}} \text { if } x_{1} \neq 1 \text { with } y_{1} \in X_{1}, \\ t_{0}^{\prime} \in T_{0} \text { such that } x_{1}^{s_{1}}=y_{1} t_{0}^{\prime} s_{1} y_{1} .\end{array}\right.$

(ii) $s_{1}\left(x_{1} x_{12} t_{0} s_{1} s_{2}\right)=\left\{\begin{array}{l}x_{12}^{s_{1}} t_{0}^{s_{1}} s_{2} \text { if } x_{1}=1 ; \\ y_{1} x_{12}^{t^{\prime-1}}\left(t_{0}^{\prime} t_{0}\right) s_{1} s_{2} y_{1}^{s_{1} t_{0} s_{1} s_{2}}\left[\left(y_{1}^{s_{1} t_{0} s_{1}}\right)^{-1},\left(x_{12}^{t_{0} s_{1}}\right)^{-1}\right]^{s_{2}} \text { if } x_{1} \neq 1 \\ \text { with } y_{1} \in X_{1}, t_{0}^{\prime} \in T_{0} \text { such that } x_{1}^{s_{1}}=y_{1} t_{0}^{\prime} s_{1} y_{1}, \\ y_{1}^{s_{1} t_{0} s_{1} s_{2}} \in X_{12} \text { and }\left[\left(y_{1}^{s_{1} t_{0} s_{1}}\right)^{-1},\left(x_{12}^{t_{0} s_{1}}\right)^{-1}\right]^{s_{2}} \in X_{1} .\end{array}\right.$ 
(iii) $s_{1}\left(x_{1} x_{2} x_{12} t_{0} s_{1} s_{2} s_{1}\right)=\left\{\begin{array}{l}x_{2}^{s_{1}} x_{12}^{s_{1}} t_{0}^{s_{1}} s_{2} s_{1} \text { if } x_{1}=1 ; \\ y_{1}\left(x_{2}\left[y_{1}^{-1},\left(x_{12}^{s_{1}}\right)^{-1}\right]^{s_{1}}\right)^{t_{0}^{\prime-1}} x_{12}^{t_{0}^{-1}}\left(t_{0}^{\prime} t_{0}\right) s_{1} s_{2} s_{1} y_{1}^{s_{1} t_{0} s_{1} s_{2} s_{1}} \\ \text { if } x_{1} \neq 1 \text { with } y_{1} \in X_{1}, t_{0}^{\prime} \in T_{0} \text { such that } \\ x_{1}^{s_{1}}=y_{1} t_{0}^{\prime} s_{1} y_{1} \quad \text { and } y_{1}^{s_{1} t_{0} s_{1} s_{2} s_{1}} \in X_{2} .\end{array}\right.$

From the above relations we obtain the action of $s_{1}$ :

$s_{1}\left(t_{0} b\right)=t_{0}^{s_{1}} s_{1} b$

$s_{1}\left(x_{1} t_{0} s_{1} b\right)=\left\{\begin{array}{l}t_{0}^{s_{1}} b \text { if } x_{1}=1 \\ \psi\left(y_{1}^{s_{1} t_{0} s_{1}}\right)\left(y_{1}\left(t_{0}^{\prime} t_{0}\right) s_{1} b\right) \text { if } x_{1} \neq 1 \text { with } y_{1} \in X_{1}, \\ t_{0}^{\prime} \in T_{0} \text { such that } x_{1}^{s_{1}}=y_{1} t_{0}^{\prime} s_{1} y_{1} .\end{array}\right.$

$s_{1}\left(x_{2} t_{0} s_{2} b\right)=x_{2}^{s_{1}} t_{0}^{s_{1}} s_{1} s_{2} b$

$s_{1}\left(x_{1} x_{12} t_{0} s_{1} s_{2} b\right)=\left\{\begin{array}{l}x_{12}^{s_{1}} t_{0}^{s_{1}} s_{2} b \text { if } x_{1}=1 ; \\ \psi\left(\left[\left(y_{1}^{s_{1} t_{0} s_{1}}\right)^{-1},\left(x_{12}^{t_{0} s_{1}}\right)^{-1}\right]^{s_{2}}\right)\left(y_{1} x_{12}^{t_{0}^{\prime-1}}\left(t_{0}^{\prime} t_{0}\right) s_{1} s_{2} b\right) \text { if } x_{1} \neq 1 \\ \text { with } y_{1} \in X_{1}, t_{0}^{\prime} \in T_{0} \text { such that } x_{1}^{s_{1}}=y_{1} t_{0}^{\prime} s_{1} y_{1} .\end{array}\right.$

$s_{1}\left(x_{2} x_{12} t_{0} s_{2} s_{1} b\right)=x_{12}^{s_{1}} x_{2}^{s_{1}} t_{0}^{s_{1}} s_{1} s_{2} s_{1} b$

$s_{1}\left(x_{1} x_{2} x_{12} t_{0} s_{1} s_{2} s_{1} b\right)=\left\{\begin{array}{l}x_{2}^{s_{1}} x_{12}^{s_{1}} t_{0}^{s_{1}} s_{2} s_{1} b \text { if } x_{1}=1 ; \\ \psi\left(y_{1}^{s_{1} t_{0} s_{1} s_{2} s_{1}}\right)\left(y_{1}\left(x_{2}\left[y_{1}^{-1},\left(x_{12}^{s_{1}}\right)^{-1}\right]^{s_{1}}\right)^{t_{0}^{\prime-1}} x_{12}^{t_{0}^{\prime-1}}\left(t_{0}^{\prime} t_{0}\right) s_{1} s_{2} s_{1} b\right) \\ \text { if } x_{1} \neq 1 \text { with } y_{1} \in X_{1}, t_{0}^{\prime} \in T_{0} \text { such that } x_{1}^{s_{1}}=y_{1} t_{0}^{\prime} s_{1} y_{1} .\end{array}\right.$

\section{The action of $s_{2}$}

For the generator $s_{2}$ we need the relations:

(i) $s_{2}\left(x_{2} t_{0} s_{2}\right)=\left\{\begin{array}{l}t_{0}^{s_{2}} \text { if } x_{2}=1 \\ y_{2}\left(t_{0}^{\prime} t_{0}\right) s_{2} y_{2}^{s_{2} t_{0} s_{2}} \text { if } x_{2} \neq 1 \text { with } y_{2} \in X_{2}, \\ t_{0}^{\prime} \in T_{0} \text { such that } x_{2}^{s_{2}}=y_{2} t_{0}^{\prime} s_{2} y_{2}\end{array}\right.$ 
(ii) $s_{2}\left(x_{2} x_{12} t_{0} s_{2} s_{1}\right)=\left\{\begin{array}{l}x_{12}^{s_{2}} t_{0}^{s_{2}} s_{1} \text { if } x_{2}=1 ; \\ y_{2} x_{12}^{t_{0}^{\prime-1}}\left(t_{0}^{\prime} t_{0}\right) s_{2} s_{1} y_{2}^{s_{2} t_{0} s_{2} s_{1}}\left[\left(y_{2}^{s_{2} t_{0} s_{2}}\right)^{-1},\left(x_{12}^{t_{0} s_{2}}\right)^{-1}\right]^{s_{1}} \text { if } x_{2} \neq 1 \\ \text { with } y_{2} \in X_{2}, t_{0}^{\prime} \in T_{0} \text { such that } x_{2}^{s_{2}}=y_{2} t_{0}^{\prime} s_{2} y_{2}, \\ y_{2}^{s_{2} t_{0} s_{2} s_{1}} \in X_{12} \text { and }\left[\left(y_{2}^{s_{2} t_{0} s_{2}}\right)^{-1},\left(x_{12}^{t_{0} s_{2}}\right)^{-1}\right]^{s_{1}} \in X_{2} .\end{array}\right.$

$\left\{\begin{array}{l}x_{1}^{s_{2}} x_{12}^{s_{2}} t_{0}^{s_{2}} s_{1} s_{2} \text { if } x_{2}=1 ; \\ {\left[y_{2}^{-1},\left(x_{12}^{s_{2}}\right)^{-1}\right]^{s_{2} t_{0}^{\prime-1}} y_{2}\left(\left[y_{2}^{-1},\left[\left(x_{12}^{s_{2}}\right)^{-1}, y_{2}^{-1}\right]^{s_{2} t_{0}^{-1}}\right] x_{1}^{s_{2}} x_{12}^{t_{0}^{\prime-1}}\right)}\end{array}\right.$

(iii) $s_{2}\left(x_{1} x_{2} x_{12} t_{0} s_{1} s_{2} s_{1}\right)=\left\{\begin{array}{l}\left(t_{0}^{\prime} t_{0}\right) s_{1} s_{2} s_{1} y_{2}^{s_{2} t_{0} s_{1} s_{2} s_{1}} \text { if } x_{2} \neq 1 \text { with } y_{2} \in X_{2}, t_{0}^{\prime} \in T_{0} \\ \text { such that } x_{2}^{s_{2}}=y_{2} t_{0}^{\prime} s_{2} y_{2},\left[y_{2}^{-1},\left(x_{12}^{s_{2}}\right)^{-1}\right]^{s_{2} t_{0}^{-1}} \in X_{1},\end{array}\right.$ $\left[y_{2}^{-1},\left[\left(x_{12}^{s_{2}}\right)^{-1}, y_{2}^{-1}\right]^{s_{2} t_{0}^{-1}}\right] x_{1}^{s_{2}} x_{12}^{t_{0}^{\prime-1}} \in X_{12}$ and $y_{2}^{s_{2} t_{0} s_{1} s_{2} s_{1}} \in X_{1}$.

These relations give the following action:

$s_{2}\left(t_{0} b\right)=t_{0}^{s_{2}} s_{2} b$

$s_{2}\left(x_{1} t_{0} s_{1} b\right)=x_{1}^{s_{2}} t_{0}^{s_{2}} s_{2} s_{1} b$

$s_{2}\left(x_{2} t_{0} s_{2} b\right)=\left\{\begin{array}{l}t_{0}^{s_{2}} b \text { if } x_{2}=1 \\ \psi\left(y_{2}^{s_{2} t_{0} s_{2}}\right)\left(y_{2}\left(t_{0}^{\prime} t_{0}\right) s_{2} b\right) \text { if } x_{2} \neq 1 \text { with } y_{2} \in X_{2}, \\ t_{0}^{\prime} \in T_{0} \text { such that } x_{2}^{s_{2}}=y_{2} t_{0}^{\prime} s_{2} y_{2} .\end{array}\right.$

$s_{2}\left(x_{1} x_{12} t_{0} s_{1} s_{2} b\right)=x_{12}^{s_{2}} x_{1}^{s_{2}} t_{0}^{s_{2}} s_{1} s_{2} s_{1} b$

$s_{2}\left(x_{2} x_{12} t_{0} s_{2} s_{1} b\right)=\left\{\begin{array}{l}x_{12}^{s_{2}} t_{0}^{s_{2}} s_{1} b \text { if } x_{2}=1 ; \\ \psi\left(\left[\left(y_{2}^{s_{2} t_{0} s_{2}}\right)^{-1},\left(x_{12}^{t_{0} s_{2}}\right)^{-1}\right]^{s_{1}}\right)\left(y_{2} x_{12}^{t_{0}^{-1}}\left(t_{0}^{\prime} t_{0}\right) s_{2} s_{1} b\right) \text { if } x_{2} \neq 1 \\ \text { with } y_{2} \in X_{2}, t_{0}^{\prime} \in T_{0} \text { such that } x_{2}^{s_{2}}=y_{2} t_{0}^{\prime} s_{2} y_{2} .\end{array}\right.$

$s_{2}\left(x_{1} x_{2} x_{12} t_{0} s_{1} s_{2} s_{1} b\right)=\left\{\begin{array}{l}x_{12}^{s_{2}} x_{1}^{s_{2}} t_{0}^{s_{2}} s_{1} s_{2} b \text { if } x_{2}=1 ; \\ \psi\left(y_{2}^{s_{2} t_{0} s_{1} s_{2} s_{1}}\right)\left(\left[y_{2}^{-1},\left(x_{12}^{s_{2}}\right)^{-1}\right]^{s_{2} t_{0}^{-1}} y_{2}\left(\left[y_{2}^{-1},\left[\left(x_{12}^{s_{2}}\right)^{-1}, y_{2}^{-1}\right]^{s_{2} t_{0}^{-1}}\right]\right.\right. \\ \left.\left.x_{1}^{s_{2}} x_{12}^{t_{0}^{\prime-1}}\right)\left(t_{0}^{\prime} t_{0}\right) s_{1} s_{2} s_{1} b\right) \text { if } x_{2} \neq 1 \text { with } y_{2} \in X_{2}, t_{0}^{\prime} \in T_{0} \\ \text { such that } x_{2}^{s_{2}}=y_{2} t_{0}^{\prime} s_{2} y_{2} .\end{array}\right.$ 


\section{References}

1. R.W. Cart er, Finite groups of Lie-type conjugacy classes and complex characters (Wiley, New York, 1985). 75, 76

2. R.W. Cart er, 'Cuspidal matrix representations for $\mathrm{GL}_{2}(q)$ and $\mathrm{GL}_{3}(q)$ ', Proc. London Math. Soc. (3) 64 (1992) 487-523. 75, 75, 75, 75, 77, 79, 83, 83, 96

3. C.W. Curt is and I. Reiner, Methods of representation theory with applications to finite groups and orders, Vol. 1 (Wiley, New York, 1981). 77, 78

4. P. Del igne and G. Lusztig, 'Representations of reductive groups over finite fields', Ann. of Math. 103 (1976) 103-161.

5. J.A. Gr een, 'The characters of the finite general linear groups', Trans. Amer. Math. Soc. 80 (1955) 402-447. 75, 96

6. C. Got sis, 'Complex representations of the general linear gGroup $G L_{n}(q)$ ', Ph.D. Thesis, Athens University (1997). 97

7. P. Fl eisch ma n $\mathrm{n}$ and I. Ja n iszczak, 'The number of regular semisimple elements for Chevalley groups of Classical type', J. of Algebra 155 (1993) 482-528. 76

8. A. Helver sen-Pasot to, 'Darstellungen von $\operatorname{GL}\left(3, \mathbf{F}_{q}\right)$ und Gaußsche Summen', Math. Ann. 260 (1982) 1-21. 75, 75

9. I. Piat et ski-Sh a pir o, 'Complex representations of $\mathrm{GL}(2, K)$ for finite fields $K$ ', Contemporary Mathematics 16 (Amer. Math. Soc., Providence, R. I, 1983). 75, 75

10. T. Springer, Seminar D, Seminar on algebraic groups and related finite groups, Lecture Notes in Mathematics 131 (ed. A. Borel et al., Springer-Verlag, Berlin, 1970). 75

D.I. Deriziotis dderiz@eudoxos.dm.uoa.gr

C. P. Gotsis cgotsis@eudoxos.dm.uoa.gr

Department of Mathematics

University of Athens

Panepistemiopolis, Athens, Greece. 